\title{
Quantum distillation of Hilbert spaces, semi-classics and anomaly matching
}

\author{
Gerald V. Dunne, ${ }^{a, b}$ Yuya Tanizaki ${ }^{a, c}$ and Mithat Ünsal ${ }^{a, d}$ \\ ${ }^{a}$ Kavli Institute for Theoretical Physics, University of California Santa Barbara, \\ CA 93106, U.S.A. \\ ${ }^{b}$ Department of Physics, University of Connecticut, \\ Storrs, CT 06269, U.S.A. \\ ${ }^{c}$ RIKEN BNL Research Center, Brookhaven National Laboratory, \\ Upton, NY 11973, U.S.A. \\ ${ }^{d}$ Department of Physics, North Carolina State University, \\ Raleigh, NC 27695, U.S.A. \\ E-mail: gerald.dunne@uconn.edu, yuya.tanizaki@riken.jp, \\ unsal.mithat@gmail.com
}

AbSTRACT: A symmetry-twisted boundary condition of the path integral provides a suitable framework for the semi-classical analysis of nonperturbative quantum field theories (QFTs), and we reinterpret it from the viewpoint of the Hilbert space. An appropriate twist with the unbroken symmetry can potentially produce huge cancellations among excited states in the state-sum, without affecting the ground states; we call this effect "quantum distillation". Quantum distillation can provide the underlying mechanism for adiabatic continuity, by preventing a phase transition under $S^{1}$ compactification. We revisit this point via the 't Hooft anomaly matching condition when it constrains the vacuum structure of the theory on $\mathbb{R}^{d}$ and upon compactification. We show that there is a precise relation between the persistence of the anomaly upon compactification, the Hilbert space quantum distillation, and the semi-classical analysis of the corresponding symmetry-twisted path integrals. We motivate quantum distillation in quantum mechanical examples, and then study its non-trivial action in QFT, with the example of the 2D Grassmannian sigma model $\operatorname{Gr}(N, M)$. We also discuss the connection of quantum distillation with large- $N$ volume independence and flavor-momentum transmutation.

KEYWORDS: Nonperturbative Effects, Sigma Models

ARXIV EPRINT: 1803.02430 


\section{Contents}

1 Introduction and sketch of the idea $\quad 1$

2 Quantum distillation and graded partition functions in QM 5

2.1 $N$-dimensional isotropic simple harmonic oscillator 5

$\begin{array}{ll}2.2 & \text { Quantum distillation and graded representations } \\ \end{array}$

2.3 Path integral interpretation: Flavor-momentum transmutation 8

2.4 Quantum distillation in $\mathbb{C P}^{N-1}$ quantum mechanics 9

3 Quantum distillation in 2d QFTs 11

3.1 Overview of the 2d bosonic $\operatorname{Gr}(N, M)$ sigma model QFT 11

$\begin{array}{lll}3.2 & \text { Hilbert space interpretation of twisted boundary conditions } & 13\end{array}$

$\begin{array}{lll}3.3 & \text { Mixed anomalies on } \mathbb{R}^{2} & 14\end{array}$

3.3.1 Mixed anomaly for bosonic $\operatorname{Gr}(N, M)$ model at $\Theta=\pi \quad 15$

3.3.2 Mixed anomaly for supersymmetric $\operatorname{Gr}(N, M)$ sigma model $\quad 17$

3.4 Adiabatic compactification and persistence of anomaly polynomial 18

$\begin{array}{ll}3.5 & \text { Overview of small- } L \text { resurgent semiclassics } \\ \end{array}$

3.6 Large- $N$ volume independence and flavor-momentum transmutation $\quad 20$

4 Conclusions: what is happening and why is it happening? 22

\section{Introduction and sketch of the idea}

In this paper, we connect the semi-classical analysis of asymptotically free quantum field theory in the calculable weak-coupling domain and with adiabatic continuity [1-4] (see also [5-29]) with the 't Hooft anomaly matching between the short distance (UV) and long distance (IR) effective theories [30,31] (see also [31-57]). We motivate our approach using ideas from graded representation theory of Hilbert space. In $2 \mathrm{~d}$ QFT, it has been shown that adiabatic circle compactification with special spatial twisted boundary conditions permits well-controlled semi-classical analysis of features such as symmetry breaking, revealing the correct theta-angle dependence (when it exists), mass gap, Borel plane and UV-IR renormalon structure. This approach applies to QFTs with instantons and theta angles, such as $O(3), \mathbb{C} \mathbb{P}^{N-1}$, and $\operatorname{Gr}(N, M)$ sigma models [2, 13, 20-22], as well as to theories without instantons or a $\Theta$-term, such as $O(N)$ with $N>3$, and the Principal Chiral Model [17, 22, 28]. In both classes of QFTs, semi-classical analysis reveals the existence of new saddles [1]. Our new Hilbert space approach gives extra physical insight into the special twisted boundary conditions and their relation to the vacuum structure of the theory in decompactification limit. In the first class of theories, those with instantons and $\Theta$-angles, 
these unique boundary conditions are the ones for which a mixed anomaly polynomial, despite being associated with a zero form symmetry, persists upon compactification.

In general, Hilbert spaces of QFTs and quantum mechanical (QM) systems are "big places". For example, even for a modest QM spin system with 50 spin- $\frac{1}{2}$ particles, the dimension of the Hilbert space is very large: $\mathcal{H}, \operatorname{dim}(\mathcal{H})=2^{50} \approx 10^{15}$. For the computation of certain properties of the system, not every state is as important as the others, as is familiar for example from the success of variational approaches to low energy and long-distance properties. In this work, motivated by ideas from graded representation theory, we analyze the structure of the Hilbert space, and try to extract the most important ground state contributions out of the vast Hilbert space. We show that this is equivalent to using the symmetry-twisted partition function instead of the thermal one. The idea is reminiscent of the supersymmetric (Witten) index [58-60], but it works equally well for non-supersymmetric theories, including purely bosonic theories. In supersymmetric systems, a natural grading is the $\mathbb{Z}_{2}$ fermionic number, $(-1)^{F}$. This grading distinguishes bosonic states from fermionic states, leading to large cancellations between degenerate states under supersymmetry, and turns a thermal state sum into a graded state-sum $\mathcal{Z}(L)=\operatorname{tr}_{\mathcal{H}}\left[\exp (-L \widehat{H})(-1)^{F}\right]$, which has no dependence on $L$. We are also motivated by the success of twisted partition functions in probing the low energy and non-perturbative properties of supersymmetric quantum field theories [61-63]. In a purely bosonic theory, or in general non-supersymmetric theories, we seek a similar operation that distills the ground state from excited states: we call this procedure quantum distillation.

As a general setup, let us consider a QFT with the global symmetry $G$, and denote its Hilbert space and Hamiltonian as $\mathcal{H}$ and $\widehat{H}$, respectively. If the symmetry $G$ is spontaneously broken to $G_{0}$, the Hilbert space is decomposed into superselection sectors labeled by the coset space $G / G_{0}$ :

$$
\mathcal{H}=\bigoplus_{v \in G / G_{0}} \mathcal{H}^{(v)}
$$

where the matrix elements of any local operators between $\mathcal{H}^{(v)}$ and $\mathcal{H}^{\left(v^{\prime}\right)}$ are zero in the infinite volume limit $\left(v \neq v^{\prime}\right)$. Since $\mathcal{H}^{(v)}$ and $\mathcal{H}^{\left(v^{\prime}\right)}$ are related by the broken symmetry $G$, they have the same energy spectrum. Assuming the absence of accidental degeneracy, energy eigenstates of each $\mathcal{H}^{(v)}$ with energy $E$ are in some irreducible representation $\mathcal{R}_{E}$ of the unbroken symmetry $G_{0}$, and especially the ground state is singlet which we denote as $\mathcal{R}_{0}$ :

$$
\mathcal{H}^{(v)} \simeq \mathcal{R}_{0} \oplus \bigoplus_{E>0} \mathcal{R}_{E}
$$

The ordinary partition function treats states within a given representation $\mathcal{R}_{E}$ exactly in the same way, since each state appears once with its Boltzmann weight:

$$
\mathcal{Z}(\beta)=\operatorname{tr}_{\mathcal{H}}[\exp (-\beta \widehat{H})]=\operatorname{vol}\left(G / G_{0}\right)\left(1+\sum_{E>0} \operatorname{dim}\left(\mathcal{R}_{E}\right)[\exp (-\beta E)]\right) .
$$

For a system with large global symmetries, $\operatorname{dim}\left(\mathcal{R}_{E}\right)$ can grow very quickly as a function of $E$. Therefore, in order to extract ground state properties, such as the ground state 
degeneracy $\operatorname{vol}\left(G / G_{0}\right)$, we have to take the limit $\beta \rightarrow \infty$, which is typically the stronglycoupled regime of interesting field theories.

Let $\widehat{Q}_{i}$ denote the generators of the maximal torus of $G_{0}$, so that $\left[\widehat{Q}_{i}, \widehat{Q}_{j}\right]=\left[\widehat{Q}_{i}, \widehat{H}\right]=0$, and we introduce the symmetry-twist operator,

$$
\widehat{\Omega}=\exp \left(\mathrm{i} \sum_{j} \alpha_{j} \widehat{Q}_{j}\right) .
$$

We define the symmetry-twisted partition function as:

$$
\mathcal{Z}_{\Omega}(L)=\operatorname{tr}_{\mathcal{H}}[\exp (-L \widehat{H}) \widehat{\Omega}]=\operatorname{vol}\left(G / G_{0}\right)\left(1+\sum_{E>0} \operatorname{tr}_{\mathcal{R}_{E}}(\widehat{\Omega}) \exp (-L E)\right) .
$$

Since $\left[\widehat{Q}_{i}, \widehat{H}\right]=0$, eigenstates of $\widehat{H}$ are also eigenstates of $\widehat{Q}_{i}$, and (1.5) provides pure phase assignments to states in each $\mathcal{R}_{E}$. These states in $\mathcal{R}_{E}$ are, by symmetries, degenerate, so the phases provide an opportunity for the states within $\mathcal{R}_{E}$ to interfere destructively, leading to state-cancellations, so that $\operatorname{tr}_{\mathcal{R}_{E}}(\widehat{\Omega}) \ll \operatorname{dim}\left(\mathcal{R}_{E}\right)$. When this is true, we can extract the ground-state properties from $\mathcal{Z}_{\Omega}(L)$ without taking $L \rightarrow \infty$, providing more useful information concerning the nonperturbative aspects of asymptotically free QFTs. ${ }^{1}$

What is this good for? There are three related answers to this question. We also summarize these viewpoints in figure 1 :

- Analyticity, adiabatic continuity, and semi-classics: to study the ground state properties in a non-trivial asymptotically free QFT, a natural approach is to take advantage of the weak coupling at small- $\beta$ (high temperature). However, at small- $\beta$, $\mathcal{Z}(\beta)$ is an extremely contaminated quantity. It has contributions from a huge tower of states with similar order of magnitude Boltzmann weights $e^{-\beta E}$. Furthermore, in the presence of phase transitions, $\mathcal{Z}(\beta)$ is non-analytic as a function of $\beta$. On the other hand, a suitable $\mathcal{Z}_{\Omega}(L)$ may be a relatively uncontaminated quantity, dominated by a few or even a single state ${ }^{2}$ due to large-cancellations in the state-sum $[2,26]$. As a result, it may be analytic as a function of $L$ and can continuously connect small and large $L$ limits. If this is the case, the corresponding path integral does not change dramatically as the radius is changed. At small- $L$ where the theory becomes weakly coupled, the corresponding path integral encodes semi-classical non-perturbative information about the vacuum structure of the theory which is adiabatically connected

\footnotetext{
${ }^{1} \mathrm{~A}$ real chemical potential does not cause a sign problem in the Hamiltonian formulation, but does create a sign problem in the Euclidean path integral formulation. The grading in (1.5) corresponds to imaginary chemical potentials. In this case, it does not create a sign problem in the Euclidean path integral formulation, but it does cause a sign "problem" in the Hamiltonian formulation (the graded state-sum). However, this Hamiltonian sign "problem" is actually useful. Thus, the physical intuition behind real and imaginary chemical potentials are somehow opposite. The former creates preference of some states over the others by altering the magnitude of their Boltzmann weight, the latter does not change the magnitude of the Boltzmann weight, but attaches a pure phase to the state according to its charge.

${ }^{2}$ In this sense, our approach has a similarity to tensor network approach to QFT [64]. Both may pick out a subset of important states in an otherwise exponentially large Hilbert space.
} 


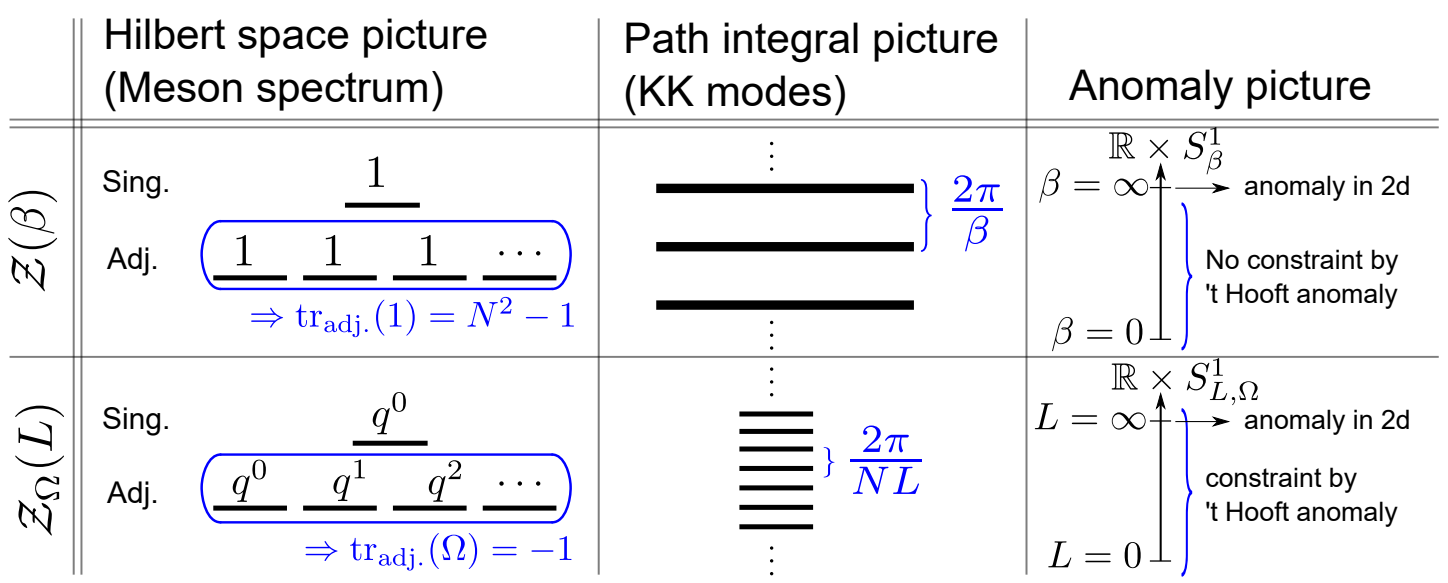

Figure 1. Schematic illustration of the advantages of the symmetry-twisted partition function $\mathcal{Z}_{\Omega}(L)$ in comparison with the thermal partition function $\mathcal{Z}(\beta)$. In the left column, we count the degrees of freedom of mesons. Mesons give a large, $O\left(N^{2}\right)$ contribution to $\mathcal{Z}(\beta)$ since they are in the adjoint representation of the $\mathrm{SU}(N) / \mathbb{Z}_{N}$ symmetry, while $\mathcal{Z}_{\Omega}(L)$ is affected only by $O(1)$. In the middle column, it is explained by the Kaluza-Klein (KK) or Matsubara decomposition of the fields, and the KK modes in $\mathcal{Z}_{\Omega}(L)$ are much denser than those of $\mathcal{Z}(\beta)$, due to the flavor-momentum transmutation. In the right column, we explain its consequence for the 't Hooft anomaly of the theory, and the 't Hooft anomaly in 2 dimensions persists in $\mathcal{Z}_{\Omega}(L)$ for any $L$, while it survives in $\mathcal{Z}(\beta)$ only for $\beta \rightarrow \infty$.

to the strong coupling regime at large- $L[1]$. Note that this construction does not change the theory, vacuum or Hilbert space. We are simply probing its low energy properties with a different operator, leading to a generalization of partition function, in which there may be potentially large cancellations between states, and which may be analytic.

- Quantum distillation and mixed anomalies: assume the quantum theory has a mixed anomaly between two symmetries $G_{1}$ and $G_{2}$. Further, assume that these are zero-form symmetries. It has recently been shown [55], and here we generalize this argument to the Grassmannian $\operatorname{Gr}(N, M)$ sigma model, that an anomaly polynomial that exists on $\mathbb{R}^{d}$ persists on $\mathbb{R}^{d-1} \times S^{1}$ if and only if one uses a unique background holonomy associated with $G_{1}$. This is equivalent to putting a unique twisted boundary condition associated with the path integral formulation of the same theory, and correspondingly a unique (ideal) distillation of the Hilbert space.

- Flavor-momentum transmutation in the path integral: when a theory is compactified on a circle of radius $L$, and one uses the special graded partition function $\mathcal{Z}_{\Omega}(L)$, the $G$ singlet observables in the theory exhibit volume independence at large $\operatorname{rank}(G)$. The Kaluza-Klein decomposition of modes in path integral involves the quantization of momentum not in units of $\frac{2 \pi}{L}$, but rather in units of $\frac{2 \pi}{L \operatorname{rank}(G)}$, for large rank. In other words, the flavor index transmutes to a momentum index. This provides the perturbative intuition behind the idea of large- $N$ volume independence $[65,66]$. 
All these features of the symmetry-twisted partition function $\mathcal{Z}_{\Omega}(L)$ provide special advantages for the study of the ground-state properties of asymptotically-free QFTs.

\section{Quantum distillation and graded partition functions in QM}

In this section, we use two simple QM examples to explain the underlying physical intuition of the graded (or symmetry-twisted) partition function. We first discuss the $N$-dimensional simple harmonic oscillator. The simplicity of this example should not deceive the reader. Since it is the global symmetry that matters for the discussion, this example illustrates some interesting effects relevant for non-trivial QFTs. Next, we discuss QM of a particle on the $\mathbb{C} \mathbb{P}^{N-1}$ manifold.

\subsection{N-dimensional isotropic simple harmonic oscillator}

Consider the $N$-dimensional isotropic simple harmonic oscillator

$$
\widehat{H}=\sum_{j=1}^{N} \frac{1}{2}\left(\widehat{p}_{j}^{2}+\widehat{x}_{j}^{2}\right)=\sum_{j=1}^{N}\left(\widehat{a}_{j}^{\dagger} \widehat{a}_{j}+\frac{1}{2}\right),
$$

which has a global $\mathrm{U}(N)$ symmetry. The canonical commutation relation is $\left[\widehat{x}_{i}, \widehat{p}_{j}\right]=$ $\mathrm{i} \delta_{i j}$, and creation and annihilation operators are introduced as $\widehat{a}_{i}=\frac{1}{\sqrt{2}}\left(\widehat{x}_{i}+\mathrm{i} \widehat{p}_{i}\right)$ and $\widehat{a}_{i}^{\dagger}=\frac{1}{\sqrt{2}}\left(\widehat{x}_{i}-\mathrm{i} \widehat{p}_{i}\right)$, respectively. The ground state $|0\rangle$ is characterized by $\widehat{a}_{i}|0\rangle=0$ for all $i=1, \ldots, N$, and the Hilbert space is spanned by the Fock basis,

$$
\left|\left\{n_{i}\right\}\right\rangle=\frac{1}{\sqrt{n_{1} ! \cdots n_{N} !}}\left(\widehat{a}_{1}^{\dagger}\right)^{n_{1}} \cdots\left(\widehat{a}_{N}^{\dagger}\right)^{n_{N}}|0\rangle .
$$

The energy of the state $\left|\left\{n_{i}\right\}\right\rangle$ is

$$
E_{\left\{n_{i}\right\}}=\frac{N}{2}+\sum_{i=1}^{N} n_{i}
$$

The global $\mathrm{U}(N)$ symmetry acts as: $\widehat{a}_{j} \mapsto U_{i j} \widehat{a}_{j}$, with $U \in \mathrm{U}(N)$. Since we consider a bosonic system, the states are classified by the totally symmetric representations of this $\mathrm{U}(N)$ symmetry. In the highest weight notation, they are denoted as $(\lambda, 0, \ldots, 0)$, and the corresponding Young tableau has one row with $\lambda$ boxes:

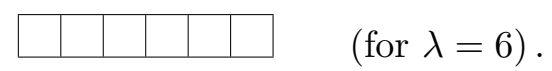

The degeneracy is the dimension of the representation:

$$
\operatorname{dim}(\lambda, 0, \ldots, 0)=\left(\begin{array}{c}
N+\lambda-1 \\
\lambda
\end{array}\right)
$$


The thermal partition function of this system is given by

$$
\begin{aligned}
\mathcal{Z}(\beta) & =\sum_{\lambda=0}^{\infty} \sum_{n_{1}, \ldots, n_{N} \geq 0} \delta_{n_{1}+\cdots+n_{N}, \lambda} \mathrm{e}^{-\beta E_{\left\{n_{i}\right\}}} \\
& =e^{-\beta N / 2} \sum_{\lambda=0}^{\infty}\left(\begin{array}{c}
N+\lambda-1 \\
\lambda
\end{array}\right) \mathrm{e}^{-\beta \lambda} \\
& =\frac{1}{(2 \sinh (\beta / 2))^{N}}
\end{aligned}
$$

The degeneracy factor grows very rapidly. In fact, in the large- $N$ limit, the states with $\lambda \sim N$ have exponentially large degeneracy. This is a quite generic feature of thermal partition functions for theories with large global symmetries.

\subsection{Quantum distillation and graded representations}

We now construct a symmetry-twisted partition function, which is free from contamination by high-energy states. Define the symmetry operator $\widehat{\Omega}$

$$
\widehat{\Omega}=\exp \left(\mathrm{i} \sum_{k=1}^{N} \alpha_{k} \widehat{Q}_{k}\right), \quad \text { where } \alpha_{k}=\frac{2 \pi}{N} k, \widehat{Q}_{k}=\widehat{a}_{k}^{\dagger} \widehat{a}_{k} .
$$

It acts on the Fock basis as

$$
\left|\left\{n_{i}\right\}\right\rangle \mapsto \exp \left(\mathrm{i} \sum_{k=1}^{\infty} \frac{2 \pi}{N} k n_{k}\right)\left|\left\{n_{i}\right\}\right\rangle .
$$

The corresponding $N \times N$ twist matrix $\Omega_{i j}$ is defined by

$$
\widehat{\Omega} \widehat{a}_{j}^{\dagger} \widehat{\Omega}^{-1}=\Omega_{i j} \widehat{a}_{j}^{\dagger},
$$

and is diagonal, with entries being powers of the $N$-th root of unity:

$$
\Omega=\operatorname{diag}\left[q, q^{2}, \ldots, q^{N}\right], \quad q \equiv \exp \left(\frac{2 \pi \mathrm{i}}{N}\right)
$$

Using this symmetry operator $\widehat{\Omega}$, we define the symmetry-twisted partition function,

$$
\mathcal{Z}_{\Omega}(L)=\operatorname{tr}[\widehat{\Omega} \exp (-L \widehat{H})] .
$$

We use the symbol $L$ instead of $\beta$, to further distinguish the twisted partition function (1.5) from the thermal partition function (1.3). Using the Fock basis for the trace, we find that

$$
\mathcal{Z}_{\Omega}(L)=e^{-L N / 2} \sum_{\lambda=0}^{\infty} \operatorname{tr}_{(\lambda, 0, \ldots, 0)}(\Omega) \mathrm{e}^{-L \lambda},
$$

where $\operatorname{tr}_{\mathcal{R}}$ means the trace in the representation $\mathcal{R}$, labelled in highest weight notation. We evaluate the density of states using the following formula for the totally symmetric representation,

$$
\operatorname{tr}_{(\lambda, 0, \ldots, 0)}(\Omega)=\sum_{k=0}^{\lambda} \frac{1}{k !} \sum_{r_{1}, \ldots, r_{k} \geq 1} \delta_{r_{1}+\cdots+r_{k}, \lambda} \frac{\operatorname{tr}_{\square}\left(\Omega^{r_{1}}\right) \cdots \operatorname{tr}_{\square}\left(\Omega^{r_{k}}\right)}{r_{1} \cdots r_{k}} .
$$




$$
\begin{aligned}
& \lambda=2 \underline{q^{1}} \frac{q^{2}}{q^{3}} \frac{q^{4}}{q^{1}} \underline{q^{1}} \underline{q^{2}} \underline{q^{3}} \underline{q^{4}} \underline{q^{2}} \underline{q^{4}} \\
& \lambda=1 \underline{q^{1}} \underline{q^{2}} \frac{q^{3}}{q^{4}} \\
& \lambda=0 \underline{q^{4}}
\end{aligned}
$$

Figure 2. Schematic image for the graded partition function (2.12) for an $N$-dimensional isotropic simple harmonic oscillator, with $N=4$, showing the representations with $\lambda=0,1,2$, corresponding to energy $E_{\lambda}=\frac{N}{2}+\lambda$. Since the phases $q^{n}=\exp (2 \pi i n / 4)$ are attached to the states, most of them cancel with one another, and we obtain the information of the ground states. We will see that a similar structure is also present in certain QFTs.

The trace in the defining representation $(1,0, \ldots, 0)=\square$ shows that

$$
\operatorname{tr}_{\square}\left(\Omega^{r}\right)=\left\{\begin{array}{cc}
N & (r=0 \bmod N), \\
0 & (r \neq 0 \bmod N) .
\end{array}\right.
$$

Using these results, we obtain ${ }^{3}$

$$
\operatorname{tr}_{(N, 0, \ldots, 0)}\left(\Omega^{r}\right)= \begin{cases}1 & (r=0 \bmod N), \\ 0 & (r \neq 0 \bmod N) .\end{cases}
$$

There is tremendous cancellation among degenerate states due to the phase factors attached to them, leading to

$$
\mathcal{Z}_{\Omega}(L)=e^{-L N / 2} \sum_{\lambda=0}^{\infty} \mathrm{e}^{-L N \lambda}=\frac{e^{-L N / 2}}{1-\mathrm{e}^{-N L}}=\frac{1}{2 \sinh (L N / 2)} .
$$

This should be compared with the thermal partition function (2.6). Note the very different behavior in the large scale limit ( $\beta$ or $L$, respectively):

$$
\begin{aligned}
\mathcal{Z}(\beta) & =e^{-\beta N / 2}\left(1+N e^{-\beta}+\cdots+\left(\begin{array}{c}
2 N-2 \\
N-1
\end{array}\right) e^{-(N-1) \beta}+\left(\begin{array}{c}
2 N-1 \\
N
\end{array}\right) e^{-N \beta}+\ldots\right) \\
\mathcal{Z}_{\Omega}(L) & =e^{-L N / 2}\left(1+0 \times e^{-L}+\cdots+0 \times e^{-(N-1) L}+1 \times e^{-N L}+\ldots\right) .
\end{aligned}
$$

Thus, the symmetry-twisted partition function of the $N$-dimensional isotropic oscillator at radius $L$ maps to the thermal partition function of a one-dimensional harmonic oscillator with the inverse temperature $N L$. Therefore, at large $N$, the symmetry-twisted partition function $\mathcal{Z}_{\Omega}(L)$ is much less contaminated by high-energy states. The relation to large $N$ volume independence in QFT is discussed in section 3.6.

This mapping, albeit simple, may in principle have dramatic implications. In particular, $N$ can be taken to infinity to reach a thermodynamic limit, and the thermal partition function of a thermal system may develop non-analyticities, and associated phase transitions. But the graded partition function maps to a system with effectively one-degree of

\footnotetext{
${ }^{3}$ Note the formula: $\sum_{k \geq 0} \frac{1}{k !} \sum_{r_{1}, \ldots, r_{k} \geq 1} \frac{\delta_{r_{1}+\cdots+r_{k}, \lambda}}{r_{1} \cdots r_{k}}=1$, coming from the Taylor expansion of $(1-x)^{-1}=$ $\exp (-\ln (1-x))$.
} 
freedom, which will not exhibit a thermodynamic phase transition. This type of simplification is what we seek in non-trivial QFTs, discussed below in section 3. This is physically interesting, because if the graded partition function is analytic as a function of $L$, that gives an opportunity to study non-perturbative aspects of asymptotically free QFTs precisely at small- $L$ where the theory becomes weakly coupled. This gives a Hilbert space reinterpretation of the phenomena of adiabatic continuity and semi-classic calculability that have been observed in $2 \mathrm{~d} \mathbb{C P}^{N-1}, O(N)$, principal chiral model, and Grassmannian sigma models $[2,13,17,18,20-22,28]$, and is also potentially related to center-stability and avoidance of Hagedorn instability in 4d QCD(adj) (QCD with multiple adjoint fermions) [1, 3$8,16,67]$. These QFTs have been analyzed from the path integral perspective. Therefore, we next briefly discuss the implications of quantum distillation of Hilbert space in the path integral formulation.

\subsection{Path integral interpretation: Flavor-momentum transmutation}

It is useful to interpret the Hilbert space distillation in the graded partition function $\mathcal{Z}_{\Omega}(L)$ from the path integral viewpoint. This provides a perspective on the volume independence phenomenon in large- $N$ QFTs. In the absence of an operator insertion to the partition function, the Kaluza-Klein modes of a spatially compactified (with radius L) QFT are quantized in units of $\frac{2 \pi}{L}$, where each KK-mode has $N$-fold degeneracy. The insertion of the operator $\widehat{\Omega}=e^{\mathrm{i} \sum \alpha_{i} Q_{i}}$ in (2.11) amounts to a finer quantization of the KK-modes, as the $N$ fold degeneracy of KK-modes is split into $N$-levels with 1-fold degeneracy, where the spacing between the levels is now $\frac{2 \pi}{L N}$. In particular, in the $L=$ fixed, $N \rightarrow \infty$ limits, the spectrum looks like the perturbative spectrum of a theory on $\mathbb{R}^{2}$, despite the fact that $L \sim O\left(N^{0}\right)$ can be arbitrarily small. We refer to this phenomenon as flavor-momentum transmutation. This is analagous to the color-momentum transmutation in the Eguchi-Kawai reductions as discussed by Gross and Kitazawa [68], and Gonzalez-Arroyo and Okawa [69-71].

More formally, in the coherent state basis, the path-integral expression of the thermal partition function of the $N$-dimensional (bosonic) oscillator is given by

$$
\mathcal{Z}(\beta)=\int_{a(\beta)=a(0)} \mathcal{D} a^{*} \mathcal{D} a \exp \left(\int_{0}^{\beta} \mathrm{d} \tau \sum_{k=1}^{N}\left[\mathrm{i} a_{k}^{*} \partial_{\tau} a_{k}-a_{k}^{*} a_{k}\right]\right),
$$

with periodic boundary conditions on the fields, as appropriate for bosonic degrees of freedom. The graded partition function can be realized with twisted boundary conditions on the $a(\tau), \mathcal{Z}_{\Omega}(L)=\int_{a(L)=\Omega a(0)}(\ldots)$. By a field redefinition, the $a(\tau)$ can be rendered periodic at the price of turning on a particular $\mathrm{SU}(N)$ background field. The result is:

$$
\mathcal{Z}_{\Omega}(L)=\int \mathcal{D} a^{*} \mathcal{D} a \exp \left(\int_{0}^{L} \mathrm{~d} \tau \sum_{k=1}^{N}\left[\mathrm{i} a_{k}^{*}\left(\partial_{\tau}+\frac{2 \pi}{N L} k\right) a_{k}-a_{k}^{*} a_{k}\right]\right) .
$$

Decomposing the field with the Matsubara modes, $a_{k}(\tau)=\frac{1}{\sqrt{L}} \sum_{m} \tilde{a}_{k, m} \mathrm{e}^{\mathrm{i} \frac{2 \pi}{L} m \tau}$, the expo- 
nent becomes

$$
\begin{aligned}
& \sum_{m=-\infty}^{\infty} \sum_{k=1}^{N}\left[\mathrm{i} \tilde{a}_{k,-m}^{*}\left(\frac{2 \pi}{L} m+\frac{2 \pi}{N L} k\right) \tilde{a}_{k, m}-\tilde{a}_{k,-m}^{*} \tilde{a}_{k, m}\right] \\
= & \sum_{m^{\prime}=-\infty}^{\infty}\left[\mathrm{i} \frac{2 \pi}{N L} m^{\prime} \tilde{a}_{-m^{\prime}}^{\prime *} \tilde{a}_{m^{\prime}}^{\prime}-\tilde{a}_{-m^{\prime}}^{\prime *} \tilde{a}_{m^{\prime}}^{\prime}\right] \\
= & \int_{0}^{N L} \mathrm{~d} \tau^{\prime}\left[\mathrm{i} a^{\prime *}\left(\tau^{\prime}\right) \partial_{\tau^{\prime}} a^{\prime}\left(\tau^{\prime}\right)-a^{\prime *}\left(\tau^{\prime}\right) a^{\prime}\left(\tau^{\prime}\right)\right],
\end{aligned}
$$

where $\tilde{a}_{m^{\prime}}^{\prime}=\tilde{a}_{k, N m}$, with $k=m^{\prime} \bmod N$. This defines the partition function of a onedimensional harmonic oscillator with inverse temperature $N L$. Because of the twisted boundary condition, the flavor-dependent imaginary chemical potential plays the role of refined Matsubara frequencies. This reproduces exactly (2.16), with the physical implications already discussed after (2.16).

\subsection{Quantum distillation in $\mathbb{C P}^{N-1}$ quantum mechanics}

As a warm-up to some $2 \mathrm{~d}$ sigma model QFTs, we now briefly consider the quantum mechanics of a particle moving on the complex projective space, $\mathbb{C P}^{N-1}$ :

$$
\mathbb{C P}^{N-1}=\frac{\mathrm{U}(N)}{\mathrm{U}(N-1) \times \mathrm{U}(1)}
$$

This QM problem is exactly solvable, and the group theoretic structure of the corresponding Hilbert space is well known: see for example [72]. One may be tempted to think that the Hilbert space again decomposes into representations of $\mathrm{U}(N)$, as in the $N$-dimensional simple harmonic oscillator discussed in the previous sub-sections. However, the correct global symmetry is in fact $\mathrm{PSU}(N)=\mathrm{SU}(N) / \mathbb{Z}_{N}$. An analogous comment applies to the familiar example of a QM particle on $S^{2}$, where we do not have half-integer spin states (in which case the symmetry would be $\mathrm{SU}(2))$ but only integer spin states, so that the faithful symmetry is $\mathrm{SU}(2) / \mathbb{Z}_{2}=\mathrm{PSU}(2)=\mathrm{SO}(3)$.

In the highest weight notation, the Hilbert space can be decomposed as [72]:

$$
\mathcal{H} \simeq \bigoplus_{\lambda=0}^{\infty} \mathcal{R}_{\lambda}
$$

with $\mathcal{R}_{\lambda}=(\lambda, \underbrace{0, \ldots, 0}_{N-3}, \lambda)$. The degeneracy of the representation grows extremely quickly:

$$
\operatorname{dim}\left(\mathcal{R}_{\lambda}\right)=\frac{2 \lambda+N-1}{N-1}\left(\begin{array}{c}
\lambda+N-2 \\
\lambda
\end{array}\right)^{2} .
$$

Therefore, the thermal partition function is:

$$
\mathcal{Z}(\beta)=\sum_{\lambda=0}^{\infty} \operatorname{dim}\left(\mathcal{R}_{\lambda}\right) \mathrm{e}^{-\beta E_{(\lambda, 0, \ldots, 0, \lambda)}},
$$


where the energy eigenvalues are expressed in terms of the quadratic Casimir:

$$
E_{(\lambda, 0, \ldots, 0, \lambda)}=2 \lambda(\lambda+N-1)
$$

Now consider the following graded partition function:

$$
\mathcal{Z}_{\Omega}=\operatorname{tr}[\widehat{\Omega} \exp (-L \widehat{H})]=\sum_{\lambda=0}^{\infty} \operatorname{tr}_{(\lambda, 0, \ldots, 0, \lambda)}(\Omega) \mathrm{e}^{-L E_{(\lambda, 0, \ldots, 0, \lambda)}}
$$

where $\Omega$ is the $\mathrm{U}(N)$ twist matrix in (2.10). This requires the knowledge of $\operatorname{tr}_{(\lambda, 0, \ldots, 0, \lambda)}(\Omega)$, which can be found easily using the multiplication identity:

$(\lambda, 0 . \cdots, 0,0) \otimes(0,0, \cdots, 0, \lambda)=(\lambda-1,0 . \cdots, 0,0) \otimes(0,0, \cdots, 0, \lambda-1)+(\lambda, 0, \cdots, 0, \lambda)$.

For example, for $\lambda=5$ and $N=4$, we have the Young tableaux expression:

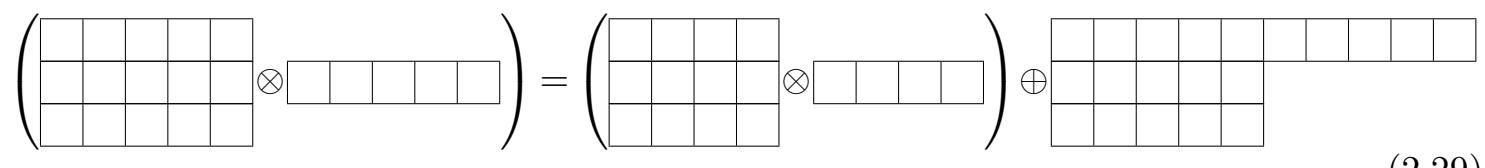

Therefore,

$$
\operatorname{tr}_{(\lambda, 0, \cdots, 0, \lambda)}(\Omega)=\left|\operatorname{tr}_{(\lambda, 0, \cdots, 0)}(\Omega)\right|^{2}-\left|\operatorname{tr}_{(\lambda-1,0, \cdots, 0)}(\Omega)\right|^{2} .
$$

This formula has several useful implications. For example, taking $\Omega=\mathbf{1}$ gives the dimension of the corresponding representation, $\operatorname{tr}_{(\lambda, 0, \cdots, 0, \lambda)}(\mathbf{1})=\operatorname{dim}((\lambda, 0, \ldots, 0, \lambda))$ :

$$
\operatorname{dim}((\lambda, 0, \ldots, 0, \lambda))=\left(\begin{array}{c}
\lambda+N-1 \\
\lambda
\end{array}\right)^{2}-\left(\begin{array}{c}
\lambda+N-2 \\
\lambda-1
\end{array}\right)^{2}=\frac{2 \lambda+N-1}{N-1}\left(\begin{array}{c}
\lambda+N-2 \\
\lambda
\end{array}\right)^{2}
$$

agreeing with the degeneracy (2.24) of the states in the representation $\mathcal{R}=(\lambda, 0, \ldots, 0, \lambda)$.

The expression (2.30) implies that it is natural to grade with respect to totally symmetric representations (as in the harmonic oscillator example), but of two different sizes. This leads to dramatic cancellations in the graded state sum (2.11). Indeed, using (2.30) and (2.15), we find that for the $\mathrm{U}(N)$ twist (2.10)

$$
\operatorname{tr}_{(\lambda, 0, \ldots, 0, \lambda)}(\Omega)=\left\{\begin{array}{cl}
1 & (\lambda=0 \bmod N) \\
-1 & (\lambda=1 \bmod N) \\
0 & \text { (others) }
\end{array}\right.
$$

Therefore, the symmetry-twisted partition function reduces to

$$
\mathcal{Z}_{\Omega}(L)=\sum_{k=0}^{\infty}\left(\mathrm{e}^{-L E_{(k N, 0, \ldots, 0, k N)}}-\mathrm{e}^{\left.-L E_{(k N+1,0, \ldots, 0, k N+1)}\right)}\right.
$$

In the large- $L$ (arbitrary $N$ ), as well as large- $N$ (arbitrary $L$ ) limits, the sum is dominated by low $k$ values:

$$
\mathcal{Z}_{\Omega}(L) \sim 1-e^{-2 N L}+0+\cdots+0+e^{-4 N(N-1 / 2) L}-e^{-4 N(N+1) L}+\ldots
$$


This should be contrasted with the thermal partition function:

$$
Z(\beta) \sim 1+\left(N^{2}-1\right) e^{-2 N \beta}+\frac{1}{4} N^{2}(N-1)(N+3) e^{-4(N+1) \beta}+\ldots
$$

We again emphasize that in obtaining $\mathcal{Z}_{\Omega}(L)$, we did not change the theory. The Hilbert space associated with the graded and thermal partition functions are one and the same. In the next section, we generalize this Hilbert space argument to QFT, and explore its connection to mixed anomalies.

\section{Quantum distillation in 2d QFTs}

\subsection{Overview of the 2d bosonic $\operatorname{Gr}(N, M)$ sigma model QFT}

Now, we move to an interesting class of QFTs, the Grassmannian $\operatorname{Gr}(N, M)$ sigma models in $2 \mathrm{~d}$. This theory has the following properties which makes it interesting in its own right, in addition to being a testing-ground for ideas in QCD-like theories [73, 74].

- Asymptotic freedom and dynamically induced mass

- Instantons on any 2 dimensional Euclidean spacetime manifold $\mathcal{M}_{2}$, and fractional instantons on $\mathbb{R} \times S_{L}^{1}$ with twisted boundary conditions.

- Confinement in the bosonic model

- Interpolation from vector model to matrix model as $M$ interpolates from $O\left(N^{0}\right)$ to $O\left(N^{1}\right)$.

- Quantum distillation in its Hilbert space with a suitably graded (symmetry-twisted) partition function.

- Theta angle and a mixed anomaly between charge-conjugation symmetry $C$, and $\operatorname{PSU}(N)$ global symmetry on $\mathbb{R}^{2}$ at $\Theta=\pi$. In the supersymmetric $\mathcal{N}=(2,2)$ extension, a mixed anomaly between discrete axial $\mathbb{Z}_{2 N}$ and $\operatorname{PSU}(N)$ global symmetry.

- Mixed anomaly (C-PSU $(N))$ on $\mathbb{R}^{1} \times S_{L}^{1}$ at $\Theta=\pi$, provided a unique twisted boundary condition is imposed associated with $\operatorname{PSU}(N)$ symmetry. ${ }^{4}$ In the supersymmetric $\mathcal{N}=(2,2)$ extension, a mixed anomaly $\left(\mathbb{Z}_{2 N}\right.$-PSU $\left.(N)\right)$, provided the same boundary conditions as in the bosonic case are used for the full super-multiplet.

Our goal in this section is to provide the relation between quantum distillation, semiclassical analysis and mixed 't Hooft anomalies. All three concepts are tied with symmetrytwisted boundary conditions in the path integral formulation and its operator image in the Hilbert space interpretation.

\footnotetext{
${ }^{4}$ Note that the anomaly polynomial and constraints remain the same as in $\mathbb{R}^{2}$, despite the fact that the associated global symmetry is zero-form. With periodic boundary conditions, the anomaly polynomial does not exist on $\mathbb{R}^{1} \times S_{L}^{1}$.
} 
Formal aspects. We first consider purely bosonic sigma models whose target space is given by the complex Grassmannian manifold $\operatorname{Gr}(N, M)[73,74]$. The elementary field is the mapping:

$$
z(x): \mathcal{M}_{2} \rightarrow \operatorname{Gr}(N, M) \equiv \frac{\mathrm{U}(N)}{\mathrm{U}(N-M) \times \mathrm{U}(M)}
$$

The real dimension of the $\operatorname{Gr}(N, M)$ space is equal to the number of microscopic degrees of freedom:

$$
\operatorname{dim}_{\mathbb{R}} \operatorname{Gr}(N, M)=N^{2}-\left[(N-M)^{2}+M^{2}\right]=2 M(N-M)
$$

Note that $M=1$ corresponds to the vector-like theory $\operatorname{Gr}(N, 1)=\mathbb{C} \mathbb{P}^{N-1}$, with dimension $2(N-1)$, while the other extreme of $M$ is the matrix-like theory $\operatorname{Gr}(2 N, N)$, with dimension $2 N^{2}$. Thus the Grassmannian models interpolate between vector-like and matrix-like behavior. This nonlinear sigma model is realized as a gauged sigma model, in terms of fields $z$ as $N \times M$ complex rectangular matrices, constrained such that $z^{\dagger} z=\mathbf{1}_{M}$. For that purpose, we first recall that

$$
\frac{\mathrm{U}(N)}{\mathrm{U}(N-M)} \simeq\left\{z \in \mathbb{C}^{N \times M} \mid z^{\dagger} z=\mathbf{1}_{M}\right\} .
$$

The action of the $\operatorname{Gr}(N, M)$ model is given by

$$
S=\frac{2}{g^{2}} \int_{M_{2}} \operatorname{tr}\left[D(a) z^{\dagger} \wedge * D(a) z\right]-\mathrm{i} \frac{\Theta}{2 \pi} \int_{M_{2}} \operatorname{tr}(F),
$$

where $z: \mathcal{M}_{2} \rightarrow \mathrm{U}(N) / \mathrm{U}(N-M), a$ is the $\mathrm{U}(M)$ gauge field on $\mathcal{M}_{2}, D(a)=\mathrm{d}+\mathrm{i} a$ is the covariant derivative, and $F=\mathrm{i}^{-1} D(a) \wedge D(a)=\mathrm{d} a+\mathrm{i} a \wedge a$ is the $\mathrm{U}(M)$ field strength. When it is evident, we simply denote $D=D(a)$. The field $z$ transforms under $\mathrm{U}(N) \times \mathrm{U}(M)$ as $z \mapsto U_{N} z U_{M}^{\dagger}$, for $\left(U_{N}, U_{M}\right) \in \mathrm{U}(N) \times \mathrm{U}(M)$ where $\mathrm{U}(N)$ is global symmetry and $\mathrm{U}(M)$ is gauge structure. The covariant derivative acts on $z$ as

$$
D z=\mathrm{d} z-\mathrm{i} z a, D z^{\dagger}=\mathrm{d} z^{\dagger}+\mathrm{i} a z^{\dagger} .
$$

Since $\operatorname{tr}(F)$ is a $\mathrm{U}(1)$ field strength, the topological charge is quantized in the integers:

$$
Q \equiv \frac{1}{2 \pi} \int_{M_{2}} \operatorname{tr}(F) \in \mathbb{Z}
$$

Thus the topological $\Theta$ angle in (3.4) is $2 \pi$ periodic, in this field normalization.

An important physical implication of the quantization of the topological charge is the existence of charge-conjugation symmetry $C$ at $\Theta \in \pi \mathbb{Z}$. Namely, the action (3.4) at $\Theta \in \pi \mathbb{Z}$ is invariant under $C$ modulo $2 \pi$, and the quantum theory is invariant under the charge conjugation. This symmetry will play a role in our discussion of quantum anomalies.

The Grassmannian model has instantons [73, 74]. The Bogomolnyi factorization of the action gives

$$
\begin{aligned}
S & =\frac{2}{g^{2}} \int \operatorname{tr}\left[(D z)^{\dagger} \wedge * D z\right] \\
& =\frac{1}{g^{2}} \int \operatorname{tr}\left[(D z \mp \mathrm{i} * D z)^{\dagger} \wedge *(D z \mp \mathrm{i} * D z)\right] \mp \mathrm{i} \frac{2}{g^{2}} \int \operatorname{tr}\left(D z^{\dagger} \wedge D z\right) \\
& =\frac{1}{g^{2}} \int \operatorname{tr}\left[(D z \mp \mathrm{i} * D z)^{\dagger} \wedge *(D z \mp \mathrm{i} * D z)\right] \pm \frac{4 \pi}{g^{2}} \int \frac{\operatorname{tr}(F)}{2 \pi} .
\end{aligned}
$$


Thus the BPS/BP S equations are $D z= \pm \mathrm{i} * D z$, and the action is bounded below, $S \geq$ $\frac{4 \pi}{g^{2}}|Q|$, for each topological sector of charge $Q$, with equality satisfied on BPS solutions.

The instanton action $S_{\mathcal{I}}$, instanton amplitude, $\Theta$-angle, dynamical strong scale $\Lambda$, and $\beta$-function are related as:

$$
S_{\mathcal{I}}=\frac{4 \pi}{g^{2}}, \quad \mathcal{I}_{2 d} \sim e^{-\frac{4 \pi}{g^{2}(\mu)}+i \Theta}, \quad \Lambda^{\beta_{0}}=\mu^{\beta_{0}} \mathcal{I}_{2 d}, \quad \text { any }(N, M)
$$

where $\beta_{0}=N$ is the leading order $\beta$-function [75]. In particular, the strong scale is related to a fractional power of the instanton amplitude, $\mathcal{I}_{2 d}^{1 / \beta_{0}} \mu=\Lambda$. Such effects play an important role in the quantum Grassmannian field theory with symmetry-twisted compactification [22].

Spectrum and representation. Let us now consider the spectrum of massive particles. As an analogy with QCD, one may consider the $z$ field as a "quark", and $z^{\dagger}$ as an "antiquark". The gauge structure of the theory is $\mathrm{U}(M)=(\mathrm{SU}(M) \times \mathrm{U}(1)) / \mathbb{Z}_{M}$, and the global symmetry is $\operatorname{PSU}(N)$. The fields $z$ and $z^{\dagger}$ would transform as fundamental $N$ and antifundamental $\bar{N}$ under the global symmetry, but these fields have both global and gauge index, and in particular, they are not gauge invariant. More precisely,

$$
z \in\left(\square_{N}, \bar{\square}_{M}\right)
$$

However, the bound states of $z$ and $z^{\dagger}$ are gauge invariant. The leading gauge invariant operators in the theory are:

$$
\left(z z^{\dagger}\right)_{j}^{k}(x) \in\left(\operatorname{Adj}_{N}, 1_{M}\right), \quad z(x)\left(e^{i \int_{x}^{y} a}\right)\left(z^{\dagger}\right)(y) \in\left(1_{N}, 1_{M}\right)
$$

which are in the adjoint and singlet representations, respectively. In the large- $N$ limit, with $M$-finite, the adjoint and singlet representations become degenerate [76].

The fact that physical states in the spectrum transform in the adjoint or product of adjoints and singlet representation (instead of the fundamental and other representations) follows from the fact that the global symmetry of the theory is not $\operatorname{SU}(N)$, but is $\operatorname{PSU}(N)=$ $\mathrm{SU}(N) / \mathbb{Z}_{N}$. In this sense, the symmetry with the faithful representations is $\operatorname{PSU}(N)$, and the same thing occurs in our $\mathbb{C} P^{N-1}$ QM example in section 2.

\subsection{Hilbert space interpretation of twisted boundary conditions}

The theory has a vacuum state, which can be described in detail semi-classically $[2,13]$. The excited states, as in the quantum mechanical example, lie in representations of $\operatorname{PSU}(N)$, and the Hilbert space admits a corresponding decomposition. The analysis is similar to the quantum mechanical $\mathbb{C} P^{N-1}$ example, except that, unlike in QM where excited singlet states do not appear in the spectrum, now one has singlet higher energy states as well, due to the existence of operators as in (3.10). Interestingly, and to a certain extent even more dramatically than in the QM examples, in the large- $N$ limit this implies that the graded state-sum in the Grassmannian QFT is even simpler. The Hilbert space construction in 
this subsection follows Sulejmanpasic [26]. Consider the insertion of the following operator into the trace:

$$
\widehat{\Omega}=e^{\mathrm{i} \sum_{k} \alpha_{k} \widehat{Q}_{k}}
$$

Using this symmetry operator $\widehat{\Omega}$, we define the symmetry-twisted partition function,

$$
\mathcal{Z}_{\Omega}(L)=\operatorname{tr}[\widehat{\Omega} \exp (-L \widehat{H})]
$$

The first excited states of the system are the adjoint and the singlet:

$$
\begin{array}{rll}
\text { adjoint : } & (1,0, \ldots, 0,1), & \text { degeneracy }=N^{2}-1 \\
\text { singlet : } & (0,0, \ldots, 0,0), & \text { degeneracy }=1
\end{array}
$$

The key facts are (2.32) and that the energy difference between these two states is $O\left(\frac{1}{N}\right)$ in the large- $N$ limit. This leads to a striking difference between the thermal state sum and the graded state sum, since these low-lying states contribute as

$$
\begin{gathered}
\mathcal{Z}(\beta) \approx+\left(N^{2}-1\right) \times e^{-\beta E_{\text {adj }}}+1 \times e^{-\beta E_{\text {singlet }}}+\ldots \underbrace{\rightarrow}_{N \rightarrow \infty} N^{2} \times e^{-\beta E_{\text {adj }}} \\
\mathcal{Z}_{\Omega}(L) \approx(-1) \times e^{-\beta E_{\text {adj }}}+1 \times e^{-\beta E_{\text {singlet }}}+\ldots \underbrace{\rightarrow}_{N \rightarrow \infty} 0
\end{gathered}
$$

Thus the important effect of the grading is to turn the $\left(N^{2}-1\right) e^{-\beta E_{\text {adj }}}$ contribution into a contribution $(-1) e^{-\beta E_{\text {adj }}}$, because of $(2.32)$. This furthermore produces a relative sign between the two leading contributions, $-1 \times e^{-\beta E_{\text {adj }}}$ and $+1 \times e^{-\beta E_{\text {singlet }}}$, in $\mathcal{Z}_{\Omega}(L)$, which leads therefore to a cancellation in the large- $N$ limit, due to the degeneracy of $E_{\text {adj }}$ and $E_{\text {singlet }}$ at large $N$. For higher energy states, for any $k$-index representation with $k \neq 0$ modulo $N, \operatorname{tr}_{\mathcal{R}}[\Omega]=0$. And the next states that can potentially contribute have masses of order $O(N)$. Therefore, we find that the graded partition function has a simple large $N$ limit:

$$
\lim _{N \rightarrow \infty} \mathcal{Z}_{\Omega}(L) \sim \mathrm{e}^{-L E_{(0,0, \ldots, 0,0)}}
$$

In particular, it is analytic as a function of $L$, and cannot exhibit a phase transition as $L$ changes.

Note that analyticity of (3.15) is in sharpe contrast with the behavior of thermal partition function, which has a deconfinement temperature $T_{d}=\frac{\Lambda}{\ln N}$, as a result of which the theory becomes deconfined at large- $N$ limit once a temperature is turned on [77]. In distinction, (3.15) is analytic as a function of $L$, and is dominated by the same state both at small and large $L$. In other words, $\beta_{\text {critical }} \rightarrow \infty$ in Affleck's study [77], while $L_{c} \rightarrow 0$ in [2].

\subsection{Mixed anomalies on $\mathbb{R}^{2}$}

In this section, we consider the constraint on the vacuum structure coming out of the 't Hooft anomaly matching. Following refs. [55, 56], we construct the 't Hooft anomaly under 
adiabatic $S^{1}$ compactification starting from that of the Grassmannian sigma model on $\mathbb{R}^{2}$. To describe the mixed anomalies, we consider two examples.

- Bosonic $\operatorname{Gr}(N, M)$ model at $\Theta=\pi$. The mixed 't Hooft anomaly ${ }^{5}$ is between PSU $(N)$ and charge conjugation symmetry $\mathrm{C}$.

- Supersymmetric $\mathcal{N}=(2,2) \operatorname{Gr}(N, M)$ model. The mixed anomaly is between $\operatorname{PSU}(N)$ and axial $\mathbb{Z}_{2 N}$ symmetry.

The reason for this exercise is two-fold. First, the anomaly has the important effect that it dramatically reduces the possibilities of IR theories. Second, despite the fact that we are considering zero-form symmetries, the anomaly polynomial persists upon compactification on $\mathbb{R} \times S_{L}^{1}$, if and only if one uses a unique twisted boundary condition, corresponding precisely to the graded partition function $\mathcal{Z}_{\Omega}(L)=\operatorname{tr}[\widehat{\Omega} \exp (-L \widehat{H})]$. In path integral language, this corresponds to a background $\mathrm{SU}(N)$ holonomy on the compactified spacetime manifold, $\mathbb{R} \times S_{L}^{1}$, as used in the semi-classical studies of these theories on $\mathbb{R} \times S^{1}[2,13,22]$. In other words, there is a precise relation between the persistence of the anomaly upon compactification, the Hilbert space quantum distillation, and the semi-classical analysis of the corresponding path integrals. We first describe the mixed anomaly, and then discuss some of the physics associated with it.

\subsubsection{Mixed anomaly for bosonic $\operatorname{Gr}(N, M)$ model at $\Theta=\pi$}

We first gauge the $\operatorname{PSU}(N)$ symmetry, and show that one loses $2 \pi$ periodicity of the $\Theta$ angle. As a result, $\mathrm{C}$ will be lost as a symmetry, implying a mixed anomaly.

To gauge the flavor symmetry, we introduce a background $\mathrm{SU}(N)$ one-form gauge field $A$, and a $\mathbb{Z}_{N}$ two-form gauge field $B$. The discrete two-form gauge structure can be realized as a pair $(B, C)$, a $\mathrm{U}(1)$ two-form gauge field $B$ and a $\mathrm{U}(1)$ one-form gauge field $C$ satisfying the constraint $N B+\mathrm{d} C=0$.

Minimal coupling gives the action:

$$
S_{\text {gauged }}=\frac{2}{g^{2}} \int_{\mathbb{R}^{2}} \operatorname{tr}\left[D(a, \widetilde{A}) z^{\dagger} \wedge * D(a, \widetilde{A}) z\right]-\mathrm{i} \frac{\Theta}{2 \pi} \int_{\mathbb{R}^{2}}[\operatorname{tr}(F)+B] .
$$

Here, $D(a, \widetilde{A}) z=\mathrm{d} z-\mathrm{i} z a+\mathrm{i} \widetilde{A} z$, and $\widetilde{A}=A+\frac{1}{N} C$ is the $\mathrm{U}(N)$ gauge field. To obtain this action, we impose the manifest $\mathrm{U}(1)$ one-form gauge invariance under

$$
B \mapsto B-\mathrm{d} \lambda, \widetilde{A} \mapsto \widetilde{A}+\lambda, \quad a \mapsto a+\lambda .
$$

By checking the spectrum of gauge-invariant line operators, we can immediately see that the faithful symmetry group $\mathrm{SU}(N) / \mathbb{Z}_{N}$ is correctly gauged [79]: if we consider the $\mathrm{U}(N)$ Wilson line in the fundamental representation, $W(C)=\operatorname{tr} \mathcal{P} \exp \left(\mathrm{i} \int_{C} \widetilde{A}\right)$, then $W(C)^{k}$ is not one-form gauge invariant for any $k \neq 0 \bmod N$. To maintain gauge invariance, we must

\footnotetext{
${ }^{5}$ It has recently been shown that the bosonic $\mathbb{C P}^{1}$ model also has a mixed 't Hooft anomaly between $\mathrm{C}$, parity $\mathrm{P}$, and time reversal $\mathrm{T}$ symmetries [78] (see also [52]). In this paper, we do not discuss this anomaly, but it is an interesting future question whether this anomaly can also persist under a certain boundary condition.
} 
attach the surface operator $\exp \left(-\mathrm{i} k \int_{S} B\right)$ with $\partial S=C$, but it depends on the topology of the surface and they are no longer genuine line operators.

A direct consequence at this stage is that the topological term (3.6) in the partition function is no longer quantized in integer units. Instead,

$$
Q=\frac{1}{2 \pi} \int_{\mathbb{R}^{2}}[\operatorname{tr}(F+B)]=\underbrace{\int_{\mathbb{R}^{2}} \frac{\mathrm{d}(\operatorname{tr}(a))}{2 \pi}}_{\in \mathbb{Z}}+\underbrace{\frac{M}{N} \int_{\mathbb{R}^{2}} \frac{\mathrm{d} C}{2 \pi}}_{\in \frac{M}{N} \mathbb{Z}} \in \frac{\operatorname{gcd}(N, M)}{N} \mathbb{Z}
$$

which implies that the $\Theta$ angle is no longer $2 \pi$ periodic, but is $2 \pi N / \operatorname{gcd}(M, N)$ periodic. Since strict $2 \pi$ periodicity was crucial for $C$ symmetry at $\Theta=\pi$, now, this symmetry is lost, implying a mixed anomaly. Before discussing the implications of this mixed anomaly, let us examine the same effect from the perspective of the partition function.

Denote the partition function in the background $(A, B)$ gauge fields as

$$
\mathcal{Z}_{\Theta}[(A, B)]=\int \mathcal{D} z^{\dagger} \mathcal{D} z \mathcal{D} a \exp \left(-S_{\text {gauged }}\right),
$$

where, in the path integral, we integrate only over the fields that are present in the microscopic theory. Applying the charge-conjugation operation to this partition function, one observes that $\mathcal{Z}$ at $\Theta=\pi$ is changed as:

$$
\mathcal{Z}_{\pi}[C \cdot(A, B)]=\mathcal{Z}_{\pi}[(A, B)] \exp \left(-\mathrm{i} M \int_{\mathbb{R}^{2}} B\right)
$$

in the presence of the background gauge field. This is of course the same effect that rendered the topological charge non-integer in (3.18). This gives the 't Hooft anomaly, or global inconsistency.

To demonstrate that this is a genuine anomaly, we must show that it is irremovable by a local counter-term in two dimensions. Gauge invariance says that such a term is given by

$$
\mathrm{i} k \int B,
$$

where $k=0,1, \ldots, N-1$. When this term is added, the $\mathrm{C}$ invariance at $\Theta=\pi$ requires

$$
2 k-M=0 \quad \text { modulo } N \text {. }
$$

Therefore, the apparent anomaly can be removed if and only if $2 k=M(\bmod N)$. Doing the same analysis at $\Theta=0$, we get $2 k=0 \bmod N$, and there is no anomaly at $\Theta=0$, e.g., by choosing $k=0$.

There are four cases. If $(N, M)=$ (even, odd), there is no solution for $k$ at $\Theta=\pi$, and there is an anomaly. If $(N, M)=$ (even, even), the solution at $\Theta=0$ is $k=0$, and at $\Theta=\pi$ is $k=M / 2$ and there is no counter-term that is good at both $\Theta=0, \pi$. This is the global inconsistency condition [31, 46, 53]. Below, we assume the vacuum at $\Theta=0$ is trivial in such cases; hence the vacuum at $\Theta=\pi$ cannot be trivial. There is a similar discussion for $(N, M)=($ odd, even $)$ and $(N, M)=($ odd, odd $)$.

As a result of the anomaly, there are three possible IR realizations:

1. $\mathrm{C} \times \operatorname{PSU}(N)$ ought to be spontaneously broken to an anomaly-free subgroup, 
2. There is a TQFT in the IR. A TQFT implies that there cannot be a trivial (unique) ground state once this theory is considered on arbitrary manifolds.

3. There is a CFT at the critical point.

Because of the $2 \mathrm{~d}$ nature of the theory, the Mermin-Wagner-Coleman theorem $[80,81]$ implies that the continuous global symmetry $\operatorname{PSU}(N)$ cannot be broken. As a result, if the second and third options do not take place, ${ }^{6}$ then the charge conjugation $C$ must be spontaneously broken.

Before discussing what happens to these anomaly considerations upon compactification of the QFT's spacetime manifold, we also describe our second example, the supersymmetric $\mathcal{N}=(2,2) \operatorname{Gr}(N, M)$.

\subsubsection{Mixed anomaly for supersymmetric $\operatorname{Gr}(N, M)$ sigma model}

The bosonic Grassmannian theory can be extended to an $\mathcal{N}=(2,2)$ supersymmetric model by introducing a Dirac Fermi field $\psi$, the fermionic partner of the $z$ field, constrained to satisfy $z^{\dagger} \psi=0_{M}$. The supersymmetric theory, apart from the $\operatorname{PSU}(N)$ symmetry, also possesses a $\mathrm{U}(1)_{A}$ symmetry at the classical level, transforming the Dirac fermion $\psi=\left(\begin{array}{c}\psi_{+} \\ \bar{\psi}_{-}\end{array}\right)$as

$$
\mathrm{U}(1)_{A}: \psi \mapsto e^{i \sigma_{3} \alpha} \psi
$$

where $\psi_{ \pm}$are the right/left movers. Under this transformation, the fermionic measure changes as

$$
\mathcal{D} \bar{\psi} \mathcal{D} \psi \mapsto \mathcal{D} \bar{\psi} \mathcal{D} \psi \exp \left(-2 \alpha \mathrm{i} \frac{N}{2 \pi} \int_{\mathbb{R}^{2}} \operatorname{tr}(\mathrm{d} a+\mathrm{i} a \wedge a)\right) .
$$

The $\mathrm{U}(1)_{A}$ symmetry has a global anomaly and is reduced to $\mathbb{Z}_{2 N}$ quantum mechanically. To see this, note that the axial charge conservation can be written as

$$
\Delta Q_{A}=(2 N) \times \frac{1}{2 \pi} \int_{\mathbb{R}^{2}} \operatorname{tr}(F) \in 2 N \mathbb{Z}
$$

as a result of integer-quantization of topological charge. Thus, (3.23) is a symmetry only when $\alpha \in \frac{2 \pi}{2 N} \mathbb{Z}$. Relatedly, note that the $2 \mathrm{~d}$ instanton amplitude is

$$
I_{2 \mathrm{~d}}=e^{-S_{I}}\left(\operatorname{tr} \psi_{-} \psi_{+}\right)^{N}
$$

and the global symmetry is just $\mathbb{Z}_{2 N}$.

Following the same analysis as in the bosonic discussion, there is a mixed anomaly between the $\operatorname{PSU}(N)$ and $\mathbb{Z}_{2 N}$ symmetries. To show this, gauge the $\operatorname{PSU}(N)$ symmetry by introducing a $\mathrm{U}(N)$ gauge field $\widetilde{A}$ and a $\mathrm{U}(1)$ two-form gauge field $B$ with the constraint

\footnotetext{
${ }^{6}$ For spin chains, it has been shown that topological order does not appear in the IR [82], and thus the topological order can be ruled out when the field theory appears as an IR description of certain spin systems. On the other hand, a CFT is possible, and indeed this is a part of the Haldane conjecture for half-integer antiferromagnetic spin chains $[83,84]$. Since they correspond to the $\mathbb{C P}^{1}$ model at $\Theta=\pi$, CFT matches the anomaly when $N=2$ and $M=1$.
} 
$N B+\mathrm{d}(\operatorname{tr}(\widetilde{A}))=0$. Under the $\mathrm{U}(1)_{A}$ transformation (3.23), the fermion measure is changed by

$$
\begin{aligned}
\mathcal{D} \bar{\psi} \mathcal{D} \psi & \mapsto \mathcal{D} \bar{\psi} \mathcal{D} \psi \exp \left[2 \alpha \mathrm{i}\left(\frac{M}{2 \pi} \int_{M_{2}} \operatorname{tr}(\mathrm{d} \widetilde{A}+\mathrm{i} \widetilde{A} \wedge \widetilde{A})-\frac{N}{2 \pi} \int_{M_{2}} \operatorname{tr}(\mathrm{d} a+\mathrm{i} a \wedge a)\right)\right] \\
& =\mathcal{D} \bar{\psi} \mathcal{D} \psi \exp \left[-2 N \alpha \mathrm{i}\left(\frac{1}{2 \pi} \int_{M_{2}} \operatorname{tr}(\mathrm{d} a+\mathrm{i} a \wedge a)+\frac{M}{2 \pi} \int_{M_{2}} B\right)\right]
\end{aligned}
$$

As a result, the axial charge non-conservation becomes

$$
\Delta Q_{A} \in 2 M \mathbb{Z}+2 N \mathbb{Z}=2 \operatorname{gcd}(N, M) \mathbb{Z} .
$$

This shows that once $\operatorname{PSU}(N)$ is gauged, $\mathbb{Z}_{2 N}$ is no longer a symmetry, reducing it all the way down to $\mathbb{Z}_{2 \operatorname{gcd}(M, N)}$ : under the $\mathbb{Z}_{2 N}$ transformation,

$$
\mathcal{Z}[(A, B)] \mapsto \mathcal{Z}[(A, B)] \exp \left(-\mathrm{i} M \int_{\mathbb{R}^{2}} B\right),
$$

and we obtain the 't Hooft anomaly.

Similar to the bosonic model, since the continuous global symmetry cannot be broken (invoking the Mermin-Wagner-Coleman theorem), the only option in the IR is spontaneous breaking of $\mathbb{Z}_{2 N}$ down to $\mathbb{Z}_{2 \operatorname{gcd}(M, N)}$ or smaller subgroups, assuming a TQFT or a CFT does not arise.

Indeed, the first option is consistent with dynamical breaking of chiral symmetry by a fermion-bilinear condensate:

$$
\left\langle\operatorname{tr} \psi_{-} \psi_{+}\right\rangle=N \Lambda e^{i \frac{2 \pi k}{N}}, \quad k=0,1, \ldots, N-1
$$

leading to $N$ isolated vacua, as well as being consistent with the supersymmetric index $I_{W}=\operatorname{tr}\left((-1)^{F}\right)=N[58,59]$.

\subsection{Adiabatic compactification and persistence of anomaly polynomial}

The graded partition function in the operator formalism (2.11), translated to the path integral formulation, corresponds to considering a path integral with symmetry twisted boundary conditions along the $S_{L}^{1}$ compactification

$$
z\left(x^{1}, x^{2}+L\right)=\Omega z\left(x^{1}, x^{2}\right),
$$

where

$$
\Omega=\operatorname{diag}\left(1, \omega, \ldots, \omega^{N-1}\right) \in \mathrm{U}(N)
$$

Equivalently, one may consider the insertion of a background holonomy $\Omega$ into the action. Under this boundary condition, we now show that the two-dimensional 't Hooft anomaly survives even after circle compactification on $\mathbb{R} \times S_{L}^{1}$ at arbitrarily size $L$ of the circle $S_{L}^{1}$.

The key observation is that the boundary conditions (3.31), or equivalently, the background holonomy (3.32), remain invariant under the intertwined combination of a centertransformation with phase $\omega$ and a $\mathbb{Z}_{N}$ cyclic permutation [55]. Neither of these individual 
transformations leave the boundary condition invariant, however, combining the center transformation with the opposite cyclic permutation leaves the boundary condition intact.

When taking this symmetry-twisted boundary condition, we have the $\mathbb{Z}_{N}$ symmetry generated by the following transformation,

$$
z \mapsto S z, \Phi \mapsto \omega^{-1} \Phi
$$

where $\Phi=\mathcal{P} \exp \left(\mathrm{i} \int_{S^{1}} a\right)$ is the $\mathrm{U}(M)$ Polyakov loop, and $S$ is the shift matrix of SU $(N)$ labels:

$$
S=\left(\begin{array}{ccccc}
0 & 1 & 0 & \cdots & 0 \\
0 & 0 & 1 & \cdots & 0 \\
\vdots & \vdots & \vdots & & \\
0 & 0 & 0 & \cdots & 1 \\
1 & 0 & 0 & \cdots & 0
\end{array}\right)
$$

We must combine these two transformations in order to keep the boundary condition invariant. $^{7}$

Since the $\mathbb{Z}_{N}$ transformation involves the transformation on the Polyakov loop, we must introduce the $\mathbb{Z}_{N}$ two-form gauge field,

$$
B=B^{(1)} \wedge L^{-1} \mathrm{~d} x^{2}
$$

when gauging this $\mathbb{Z}_{N}$ symmetry. Here, $B^{(1)}$ is a $\mathbb{Z}_{N}$ one-form gauge field. As a result, we obtain

$$
\mathcal{Z}_{\pi}\left[\mathrm{C} \cdot B^{(1)}\right]=\mathcal{Z}_{\pi}\left[B^{(1)}\right] \exp \left(-\mathrm{i} M \int B^{(1)}\right) .
$$

This gives the 't Hooft anomaly or global inconsistency condition on $\mathbb{R} \times S_{L}^{1}$ under the symmetry-twisted boundary condition, with the same implications as those discussed for the theory on $\mathbb{R}^{2}$.

An almost identical consideration also shows that in the $\mathcal{N}=(2,2)$ supersymmetric $\operatorname{Gr}(N, M)$ model, the anomaly remains essentially the same between $\operatorname{PSU}(N)$ and $\mathbb{Z}_{2 N}$, the discrete axial symmetry, upon compactification of the theory on $\mathbb{R}^{1} \times S_{L}^{1}$.

\subsection{Overview of small- $L$ resurgent semiclassics}

The non-perturbative dynamics of the $\mathbb{C P}^{N-1}$ and $\operatorname{Gr}(N, M)$ models with $\Omega$-twisted boundary condition has been examined in detail in the recent literature [2, 13, 20-22]. Here we highlight some of the connections with the analysis of this current paper.

As discussed earlier, $\operatorname{Gr}(N, M)$ admits instanton solutions with topological charge $Q_{T}=1$. In the presence of the $\Omega$-background, (3.8), these $2 \mathrm{~d}$ instantons split into $N$ minimal action kink-instantons, each with topological charge $Q_{T}=\frac{1}{N}[2,86-88]$. Note that in the study of Affleck, where $\Omega=1$, fractionalization to $N$ kink-instanton does not take place [77]. The fractionalization is similar to $4 \mathrm{~d}$ instantons splitting into $N$ monopole

\footnotetext{
${ }^{7}$ This is parallel to the emergence of the color-flavor center symmetry in $\mathrm{SU}\left(N_{f}\right)$-symmetric QCD with $N_{f}$ flavor of massive fermions with $\operatorname{gcd}\left(N_{f}, N\right) \geq 2[55,56,85]$.
} 
instantons in the presence of a non-trivial holonomy in gauge theories on $\mathbb{R}^{3} \times S_{L}^{1}$. The kink-instanton and anti-instanton amplitudes are given by

$$
\mathcal{K}_{j, k}=e^{-S_{I} / N} e^{i \frac{\Theta+2 \pi k}{N}}=e^{-\frac{4 \pi}{g^{2} N}} e^{i \frac{\Theta+2 \pi k}{N}}, \quad \overline{\mathcal{K}}_{j, k}=\mathcal{K}_{j, k}^{*}
$$

for each $j=0,1, \ldots, N-1$, and $k=1, \ldots, N$ is a branch label. As asserted, the $2 \mathrm{~d}$ instanton amplitude is a composite of the $N$-kink instantons: ${ }^{8}$

$$
\mathcal{I}=\prod_{j=0}^{N-1} \mathcal{K}_{j, k}=e^{-S_{I}} e^{i \Theta}=e^{-\frac{4 \pi}{g^{2}}+i \Theta}
$$

For example, the mass gap in the system is generated by the proliferation of kink-instantons, and is given by $[1,13]$

$$
m_{g}(\Theta) \propto \max _{k}\left(\mathcal{K}_{j, k}+\overline{\mathcal{K}}_{j, k}\right)=\Lambda \max _{k} \cos \left(\frac{\Theta+2 \pi k}{N}\right), \quad \Lambda=L^{-1} e^{-\frac{4 \pi}{N g^{2}\left(L^{-1}\right)}}
$$

This formula is rather intriguing. It shows that

- The mass gap may be induced by semi-classical instantons with action $S_{\mathcal{K}}=S_{\mathcal{I}} / N$.

- These semi-classical effects survive the large- $N$ limit, $e^{-\frac{4 \pi}{g^{2} N}} \sim O\left(N^{0}\right)$, unlike the $2 \mathrm{~d}$ instanton, which is suppressed as $e^{-\frac{4 \pi}{g^{2}}} \sim e^{-N}$.

- The result is multi-branched, and the choice associated with the vacuum of the theory is non-analytic at $\Theta=\pi$, related to spontaneous C-breaking and the existence of two vacua.

The scenario with two vacua that we find in the semi-classical domain is one of the possible outcomes of the anomaly consideration for these models at $\Theta=\pi$. It is also interesting to see that for $N=2, M=1$, namely the $\mathbb{C P}^{1}$ model, the mass gap vanishes at $\Theta=\pi$, at leading order in semi-classics. On $\mathbb{R}^{2}$, this is a part of the Haldane conjecture [83, 84, 89-91]. This is consistent with the CFT possibility arising from the 't Hooft anomaly matching argument.

In section 4, we explain the relation between the semi-classical analysis, and why it works the way it does on $\mathbb{R} \times S_{L}^{1}$ while also producing some of the non-perturbative aspects of the theory on $\mathbb{R}^{2}$, from the point of view of the Hilbert space quantum distillation and mixed anomalies.

\subsection{Large- $N$ volume independence and flavor-momentum transmutation}

Turning on the $\Omega$-background ensures that the anomaly polynomial survives upon compactification. There is another important property associated with this background. In the large- $N$ limit, $\mathrm{SU}(N)$ singlet observables as well as their correlation functions become

\footnotetext{
${ }^{8} \mathrm{~A}$ similar splitting formula for saddle contributions applies for other $2 \mathrm{~d}$ sigma models, such as the principal chiral model and the $O(N)$ model with $N>3$; note that these are models without instantons [17, $18,22]$.
} 
independent of $N$ for any finite value of $L$. Let us explicitly show it by using the technique developed by Sulejmanpasic [26].

The perturbative intuition behind volume independence is the following. If one imposes a trivial $\Omega=1$ background, then Kaluza-Klein momenta are naturally quantized in units of $\frac{2 \pi}{L}$. In the infinite volume limit, $L \rightarrow \infty$, the momentum modes become a continuum, producing perturbation theory on $\mathbb{R}^{2}$. However, with the non-trivial twist- $\Omega$, the momentum modes are quantized in a much finer spectrum, in units of $\frac{2 \pi}{L N}$. Thus the flavor background $\Omega$ transmutes into fractionalized momenta. In this case, to obtain perturbation theory on $\mathbb{R}^{2}$, at least, the planar perturbation theory, there are two options. i) $N=$ fixed, $L \rightarrow \infty$ as before or ii) $L=$ fixed, $N \rightarrow \infty$. In this latter case, one can derive global symmetry singlet observables by using reduced QM system. For example, the renormalization group $\beta$-function of QFT can be derived from reduced QM, by using a similar construction of the matrix model derivation of the $\beta$ function of Yang-Mills by Gross and Kitazawa [68].

Technically, the construction works as follows (see [26] for the $\mathbb{C P}^{N-1}$ argument). We fix $M$ and take the large- $N$ limit of the $\operatorname{Gr}(N, M)$ model. For simplicity, we take $\Theta=0$, but it can easily be incorporated. To take the large- $N$ limit, we define the 't Hooft coupling by

$$
2 f_{0}=g^{2} N
$$

The action becomes

$$
S_{\text {eff }}=\frac{N}{f_{0}} \int_{\mathcal{M}_{2}} \mathrm{~d}^{2} x \operatorname{tr}\left\{D_{\mu} z^{\dagger} D_{\mu} z+\mathrm{i} \lambda\left(z^{\dagger} z-1\right)\right\},
$$

where we introduced the Lagrange multiplier field $\lambda$, which is a real $M \times M$ matrixvalued field.

We consider the circle compactification $\mathcal{M}_{2}=\mathbb{R} \times S_{L}^{1}$, and we take the symmetry twisted boundary condition along $S_{L}^{1}$. Integrating out $z$ and $z^{\dagger}$, we obtain the effective action

$$
S_{\text {eff }}\left(a_{\mu}, \lambda\right)=-\operatorname{Tr} \ln \left[-D_{\mu}^{2}+\mathrm{i} \lambda\right]-\frac{\mathrm{i} N}{f_{0}} \int \mathrm{d}^{2} x \operatorname{tr}(\lambda) .
$$

We evaluate the functional trace using a plane wave basis:

$$
\begin{aligned}
& \operatorname{Tr} \ln \left[-D_{\mu}^{2}+\mathrm{i} \lambda\right] \\
= & \int \mathrm{d}^{2} x\left(\sum_{s=0}^{N-1} \frac{1}{L} \sum_{n \in \mathbb{Z}} \int \frac{\mathrm{d} k_{1}}{2 \pi} \operatorname{tr} \ln \left[-\left(D_{1}+\mathrm{i} k_{1}\right)^{2}-\left(D_{2}+\mathrm{i} \frac{2 \pi n+(2 \pi s / N)}{L}\right)^{2}+\mathrm{i} \lambda\right]\right) \\
= & N \int \mathrm{d}^{2} x\left(\frac{1}{N L} \sum_{m \in \mathbb{Z}} \int \frac{\mathrm{d} k_{1}}{2 \pi} \operatorname{tr} \ln \left[-\left(D_{1}+\mathrm{i} k_{1}\right)^{2}-\left(D_{2}+\mathrm{i} \frac{2 \pi m}{N L}\right)^{2}+\mathrm{i} \lambda\right]\right),
\end{aligned}
$$

where in the intermediate step, the sum over Kaluza-Klein modes and flavor modes merges to a much finer sum. As a result of this transmutation, in the large- $N$ limit, the explicit $L$ dependence in the integrand of $\int \mathrm{d}^{2} x$ disappears [26]:

$$
\lim _{N \rightarrow \infty}(\ldots)=\int \frac{\mathrm{d}^{2} k}{(2 \pi)^{2}} \operatorname{tr} \ln \left[-\left(D_{\mu}+\mathrm{i} k_{\mu}\right)^{2}+\mathrm{i} \lambda\right] .
$$


Therefore, we obtain the effective action as $S_{\text {eff }}=N s_{\text {eff }}\left(a_{\mu}, \lambda\right)$, where $s_{\text {eff }}\left(a_{\mu}, \lambda\right)$ is $N$ independent, and hence the action is suitable for saddle point analysis in the $N \rightarrow \infty$ limit. The effective action is identical to the one on $\mathbb{R}^{2}$ except that in the $\int_{\mathbb{R}^{2}}$ integral, the domain is now $\mathcal{M}_{2}=\mathbb{R} \times S_{L}^{1}$. This is immaterial for the saddle point analysis. Studying the gap equation in the large- $N$ analysis, one finds $a_{\mu}=0, \lambda=m_{g}^{2}[76]$. Here, $m_{g}=\mu e^{-\frac{4 \pi}{g^{2}(\mu) N}}$ has an interpretation as the mass of $z$ quanta, now derived from reduced model. This argument proves the large- $N$ volume independence in the $\Omega$-background.

The analysis is valid for any $\mathrm{SU}(N)$ invariant operators, and thus the large- $N$ scaling of correlation functions of the topological charge can also be obtained. This recovers the $\Theta$ dependence of the vacuum for large $N$, as well as the adiabatic continuity of the vacuum structure.

\section{Conclusions: what is happening and why is it happening?}

The interpretation of what is going on both in the Hilbert space and path integral formulation of these theories is actually quite intriguing. Consider the following graded state-sums, and their path integral realizations:

$$
\begin{aligned}
\mathcal{Z}_{\Omega}(L) & =\operatorname{tr}[\widehat{\Omega} \exp (-L \widehat{H})] \\
& =\int_{z\left(x^{1}, x^{2}+L\right)=\Omega z\left(x^{1}, x^{2}\right)} D z D z^{\dagger} D a e^{-S[z, \bar{z}, a]} \\
& =\int_{z\left(x^{1}, x^{2}\right)=z\left(x^{1}, x^{2}\right)} D z D z^{\dagger} D a e^{-S[z, \bar{z}, a, \Omega]}
\end{aligned}
$$

In the last step we used a field redefinition to replace $\Omega$-twisted boundary condition with $\Omega$-holonomy. Several observations are in order:

- Neither the Hilbert space of the theory nor the degeneracies associated with states are altered in our graded construction. Rather, we consider a graded state-sum with the insertion of $\widehat{\Omega}$ into the partition function, $\operatorname{tr}[\widehat{\Omega} \exp (-L \widehat{H})]$. We call this procedure quantum distillation. The distillation effectively picks out a subset of states, without applying any projections. The graded state-sum has no simple thermal interpretation (at least associated with the original theory).

- In the presence of large global symmetries, quantum distillation can in principle overwhelm exponential Hagedorn growth in the Hilbert space. In this sense, the graded partition function $\mathcal{Z}_{\Omega}(L)$ has a much stronger change of being an analytic function of $L$, with the possibility of no phase transition as $L$ changes from small to large $L$.

- Due to asymptotic freedom, the small- $L$ regime becomes weakly coupled and semiclassically accessible. What is learned on small- $L$ is not detached from $\mathbb{R}^{2}$. In circumstances where $\mathcal{Z}_{\Omega}(L)$ is an analytic function of $L$, the ground states of these two regimes are guaranteed to be continuously connected. This is the idea of adiabatic continuity. 
- Anomaly matching implies that there are three possibilities in these theories in the IR, when the Coleman-Mermin-Wagner theorem is taken into account: spontaneously broken discrete symmetry, TQFT or CFT. This anomaly consideration in principle permits a phase transition between these possibilities. But provided $\mathcal{Z}_{\Omega}(L)$ is an analytic function of $L$, what takes place at weak coupling at small- $L$ also takes place at strong coupling at large- $L$.

- In the large- $N$ limit, we can prove volume independence for observables provided they are measured using $\mathcal{Z}_{\Omega}(L)$. The fact that volume independence works implies that a distillation in the Hilbert space may even overcome exponential growth in the density of states.

- A similar graded Hilbert space analysis should apply to other $2 \mathrm{~d}$ asymptotically free sigma models, such as the principal chiral model, and the $O(N)$ sigma model.

The overall construction suggests the appealing possibility for analyticity of the graded partition function with important implications for non-perturbative properties of $2 \mathrm{~d}$ QFT in the decompactification limit.

\section{Acknowledgments}

We are grateful to Aleksey Cherman, David Gross, Tatsuhiro Misumi, Misha Shifman, Tin Sulejmanpasic, Sergei Gukov, Wolfgang Lerche, David Tong and Arkady Vainshtein for discussions. All three authors thank the KITP at Santa Barbara for its hospitality during the program "Resurgent Asymptotics in Physics and Mathematics" where this work was done. Research at KITP is supported by the National Science Foundation under Grant No. NSF PHY-1125915. The work of Y. T. is supported by the RIKEN Special Postdoctoral Researcher Program. This material is based upon work supported by the U.S. Department of Energy, Office of Science, Office of High Energy Physics under Award Number DESC0010339 (GD), and by the U.S. Department of Energy, Office of Science, Office of Nuclear Physics under Award Number DE-FG02-03ER41260 (MU).

Open Access. This article is distributed under the terms of the Creative Commons Attribution License (CC-BY 4.0), which permits any use, distribution and reproduction in any medium, provided the original author(s) and source are credited.

\section{References}

[1] G.V. Dunne and M. Ünsal, New Nonperturbative Methods in Quantum Field Theory: From Large-N Orbifold Equivalence to Bions and Resurgence, Ann. Rev. Nucl. Part. Sci. 66 (2016) 245 [arXiv: 1601.03414] [InSPIRE].

[2] G.V. Dunne and M. Ünsal, Resurgence and Trans-series in Quantum Field Theory: The $C P^{N-1}$ Model, JHEP 11 (2012) 170 [arXiv:1210.2423] [INSPIRE].

[3] M. Ünsal, Magnetic bion condensation: A new mechanism of confinement and mass gap in four dimensions, Phys. Rev. D 80 (2009) 065001 [arXiv:0709.3269] [INSPIRE]. 
[4] M. Ünsal and L.G. Yaffe, Center-stabilized Yang-Mills theory: Confinement and large N volume independence, Phys. Rev. D 78 (2008) 065035 [arXiv:0803.0344] [INSPIRE].

[5] M. Ünsal, Abelian Duality, Confinement, and Chiral-Symmetry Breaking in a SU(2) QCD-Like Theory, Phys. Rev. Lett. 100 (2008) 032005 [arXiv:0708.1772] [INSPIRE].

[6] P. Kovtun, M. Ünsal and L.G. Yaffe, Volume independence in large $N_{c}$ QCD-like gauge theories, JHEP 06 (2007) 019 [hep-th/0702021] [INSPIRE].

[7] M. Shifman and M. Ünsal, QCD-like Theories on $R_{3} \times S_{1}: A$ Smooth Journey from Small to Large $r\left(S_{1}\right)$ with Double-Trace Deformations, Phys. Rev. D 78 (2008) 065004 [arXiv: 0802.1232] [INSPIRE].

[8] M. Shifman and M. Ünsal, Multiflavor $Q C D^{*}$ on $R_{3} \times S_{1}$ : Studying Transition From Abelian to Non-Abelian Confinement, Phys. Lett. B 681 (2009) 491 [arXiv:0901.3743] [INSPIRE].

[9] G. Cossu and M. D'Elia, Finite size phase transitions in QCD with adjoint fermions, JHEP 07 (2009) 048 [arXiv:0904.1353] [INSPIRE].

[10] G. Cossu, H. Hatanaka, Y. Hosotani and J.-I. Noaki, Polyakov loops and the Hosotani mechanism on the lattice, Phys. Rev. D 89 (2014) 094509 [arXiv:1309.4198] [INSPIRE].

[11] P.C. Argyres and M. Ünsal, The semi-classical expansion and resurgence in gauge theories: new perturbative, instanton, bion and renormalon effects, JHEP 08 (2012) 063 [arXiv: 1206.1890] [INSPIRE].

[12] P. Argyres and M. Ünsal, A semiclassical realization of infrared renormalons, Phys. Rev. Lett. 109 (2012) 121601 [arXiv:1204.1661] [INSPIRE].

[13] G.V. Dunne and M. Ünsal, Continuity and Resurgence: towards a continuum definition of the $\mathbb{C P}(N-1)$ model, Phys. Rev. D 87 (2013) 025015 [arXiv:1210.3646] [inSPIRE].

[14] E. Poppitz, T. Schäfer and M. Ünsal, Continuity, Deconfinement and (Super) Yang-Mills Theory, JHEP 10 (2012) 115 [arXiv:1205.0290] [INSPIRE].

[15] M.M. Anber, S. Collier, E. Poppitz, S. Strimas-Mackey and B. Teeple, Deconfinement in $\mathcal{N}=1$ super Yang-Mills theory on $\mathbb{R}^{3} \times \mathbb{S}^{1}$ via dual-Coulomb gas and "affine" XY-model, JHEP 11 (2013) 142 [arXiv:1310.3522] [INSPIRE].

[16] G. Basar, A. Cherman, D. Dorigoni and M. Ünsal, Volume Independence in the Large N Limit and an Emergent Fermionic Symmetry, Phys. Rev. Lett. 111 (2013) 121601 [arXiv: 1306.2960] [INSPIRE].

[17] A. Cherman, D. Dorigoni, G.V. Dunne and M. Ünsal, Resurgence in Quantum Field Theory: Nonperturbative Effects in the Principal Chiral Model, Phys. Rev. Lett. 112 (2014) 021601 [arXiv: 1308.0127] [INSPIRE].

[18] A. Cherman, D. Dorigoni and M. Ünsal, Decoding perturbation theory using resurgence: Stokes phenomena, new saddle points and Lefschetz thimbles, JHEP 10 (2015) 056 [arXiv: 1403.1277] [INSPIRE].

[19] T. Misumi and T. Kanazawa, Adjoint $Q C D$ on $\mathbb{R}^{3} \times S^{1}$ with twisted fermionic boundary conditions, JHEP 06 (2014) 181 [arXiv:1405.3113] [INSPIRE].

[20] T. Misumi, M. Nitta and N. Sakai, Neutral bions in the $\mathbb{C} P^{N-1}$ model, JHEP 06 (2014) 164 [arXiv: 1404.7225] [INSPIRE].

[21] T. Misumi, M. Nitta and N. Sakai, Classifying bions in Grassmann $\sigma$-models and non-Abelian gauge theories by D-branes, PTEP 2015 (2015) 033B02 [arXiv: 1409.3444] [INSPIRE]. 
[22] G.V. Dunne and M. Ünsal, Resurgence and Dynamics of $O(N)$ and Grassmannian $\sigma$-models, JHEP 09 (2015) 199 [arXiv: 1505.07803] [INSPIRE].

[23] T. Misumi, M. Nitta and N. Sakai, Non-BPS exact solutions and their relation to bions in $\mathbb{C} P^{N-1}$ models, JHEP 05 (2016) 057 [arXiv: 1604.00839] [INSPIRE].

[24] A. Cherman, T. Schäfer and M. Ünsal, Chiral Lagrangian from Duality and Monopole Operators in Compactified QCD, Phys. Rev. Lett. 117 (2016) 081601 [arXiv:1604.06108] [INSPIRE].

[25] T. Fujimori, S. Kamata, T. Misumi, M. Nitta and N. Sakai, Nonperturbative contributions from complexified solutions in $\mathbb{C} P^{N-1}$ models, Phys. Rev. D 94 (2016) 105002 [arXiv: 1607.04205] [INSPIRE].

[26] T. Sulejmanpasic, Global Symmetries, Volume Independence and Continuity in Quantum Field Theories, Phys. Rev. Lett. 118 (2017) 011601 [arXiv:1610.04009] [InSPIRE].

[27] M. Yamazaki and K. Yonekura, From $4 d$ Yang-Mills to $2 d \mathbb{C P}^{N-1}$ model: IR problem and confinement at weak coupling, JHEP 07 (2017) 088 [arXiv: 1704.05852] [INSPIRE].

[28] P.V. Buividovich and S.N. Valgushev, Lattice study of continuity and finite-temperature transition in two-dimensional $\mathrm{SU}(N) \times \mathrm{SU}(N)$ Principal Chiral Model, arXiv:1706.08954 [INSPIRE].

[29] K. Aitken, A. Cherman, E. Poppitz and L.G. Yaffe, QCD on a small circle, Phys. Rev. D 96 (2017) 096022 [arXiv: 1707.08971] [InSPIRE].

[30] G. 't Hooft, Naturalness, chiral symmetry, and spontaneous chiral symmetry breaking, in Recent Developments in Gauge Theories. Proceedings, Nato Advanced Study Institute, Cargese, France, August 26 - September 8, 1979, vol. 59, pp. 135-157.

[31] D. Gaiotto, A. Kapustin, Z. Komargodski and N. Seiberg, Theta, Time Reversal and Temperature, JHEP 05 (2017) 091 [arXiv: 1703. 00501] [inSPIRE].

[32] Y. Frishman, A. Schwimmer, T. Banks and S. Yankielowicz, The Axial Anomaly and the Bound State Spectrum in Confining Theories, Nucl. Phys. B 177 (1981) 157 [InSPIRE].

[33] S.R. Coleman and B. Grossman, 't Hooft's Consistency Condition as a Consequence of Analyticity and Unitarity, Nucl. Phys. B 203 (1982) 205 [InSPIRE].

[34] A. Vishwanath and T. Senthil, Physics of three dimensional bosonic topological insulators: Surface Deconfined Criticality and Quantized Magnetoelectric Effect, Phys. Rev. X 3 (2013) 011016 [arXiv: 1209.3058] [INSPIRE].

[35] X.-G. Wen, Classifying gauge anomalies through symmetry-protected trivial orders and classifying gravitational anomalies through topological orders, Phys. Rev. D 88 (2013) 045013 [arXiv: 1303.1803] [INSPIRE].

[36] A. Kapustin and R. Thorngren, Anomalies of discrete symmetries in three dimensions and group cohomology, Phys. Rev. Lett. 112 (2014) 231602 [arXiv:1403.0617] [INSPIRE].

[37] A. Kapustin and R. Thorngren, Anomalies of discrete symmetries in various dimensions and group cohomology, arXiv:1404.3230 [INSPIRE].

[38] G.Y. Cho, J.C.Y. Teo and S. Ryu, Conflicting Symmetries in Topologically Ordered Surface States of Three-dimensional Bosonic Symmetry Protected Topological Phases, Phys. Rev. B 89 (2014) 235103 [arXiv: 1403.2018] [INSPIRE]. 
[39] J.C. Wang, Z.-C. Gu and X.-G. Wen, Field theory representation of gauge-gravity symmetry-protected topological invariants, group cohomology and beyond, Phys. Rev. Lett. 114 (2015) 031601 [arXiv: 1405.7689] [INSPIRE].

[40] E. Witten, Fermion Path Integrals And Topological Phases, Rev. Mod. Phys. 88 (2016) 035001 [arXiv: 1508.04715] [INSPIRE].

[41] N. Seiberg and E. Witten, Gapped Boundary Phases of Topological Insulators via Weak Coupling, PTEP 2016 (2016) 12C101 [arXiv: 1602.04251] [INSPIRE].

[42] E. Witten, The "Parity" Anomaly On An Unorientable Manifold, Phys. Rev. B 94 (2016) 195150 [arXiv: 1605.02391] [INSPIRE].

[43] Y. Tachikawa and K. Yonekura, On time-reversal anomaly of $2+1 d$ topological phases, PTEP 2017 (2017) 033B04 [arXiv: 1610.07010] [INSPIRE].

[44] Y. Tachikawa and K. Yonekura, More on time-reversal anomaly of $2+1 d$ topological phases, Phys. Rev. Lett. 119 (2017) 111603 [arXiv:1611.01601] [INSPIRE].

[45] C. Wang, A. Nahum, M.A. Metlitski, C. Xu and T. Senthil, Deconfined quantum critical points: symmetries and dualities, Phys. Rev. X 7 (2017) 031051 [arXiv:1703.02426] [INSPIRE].

[46] Y. Tanizaki and Y. Kikuchi, Vacuum structure of bifundamental gauge theories at finite topological angles, JHEP 06 (2017) 102 [arXiv:1705.01949] [INSPIRE].

[47] Z. Komargodski, A. Sharon, R. Thorngren and X. Zhou, Comments on Abelian Higgs Models and Persistent Order, arXiv:1705.04786 [INSPIRE].

[48] Z. Komargodski, T. Sulejmanpasic and M. Ünsal, Walls, anomalies and deconfinement in quantum antiferromagnets, Phys. Rev. B 97 (2018) 054418 [arXiv: 1706. 05731] [INSPIRE].

[49] G.Y. Cho, S. Ryu and C.-T. Hsieh, Anomaly Manifestation of Lieb-Schultz-Mattis Theorem and Topological Phases, Phys. Rev. B 96 (2017) 195105 [arXiv:1705.03892] [INSPIRE].

[50] H. Shimizu and K. Yonekura, Anomaly constraints on deconfinement and chiral phase transition, Phys. Rev. D 97 (2018) 105011 [arXiv:1706.06104] [INSPIRE].

[51] J. Wang, X.-G. Wen and E. Witten, Symmetric Gapped Interfaces of SPT and SET States: Systematic Constructions, arXiv:1705.06728 [INSPIRE].

[52] M.A. Metlitski and R. Thorngren, Intrinsic and emergent anomalies at deconfined critical points, arXiv: 1707.07686 [INSPIRE].

[53] Y. Kikuchi and Y. Tanizaki, Global inconsistency, 't Hooft anomaly and level crossing in quantum mechanics, PTEP 2017 (2017) 113B05 [arXiv: 1708.01962] [INSPIRE].

[54] D. Gaiotto, Z. Komargodski and N. Seiberg, Time-reversal breaking in $Q C D_{4}$, walls and dualities in $2+1$ dimensions, JHEP 01 (2018) 110 [arXiv:1708.06806] [INSPIRE].

[55] Y. Tanizaki, T. Misumi and N. Sakai, Circle compactification and 't Hooft anomaly, JHEP 12 (2017) 056 [arXiv: 1710.08923] [inSPIRE].

[56] Y. Tanizaki, Y. Kikuchi, T. Misumi and N. Sakai, Anomaly matching for the phase diagram of massless $\mathbb{Z}_{N}-Q C D$, Phys. Rev. D 97 (2018) 054012 [arXiv:1711.10487] [InSPIRE].

[57] M. Guo, P. Putrov and J. Wang, Time reversal, $\mathrm{SU}(N)$ Yang-Mills and cobordisms: Interacting topological superconductors/insulators and quantum spin liquids in $3+1 D$, Annals Phys. 394 (2018) 244 [arXiv:1711.11587] [INSPIRE]. 
[58] E. Witten, Constraints on Supersymmetry Breaking, Nucl. Phys. B 202 (1982) 253 [INSPIRE].

[59] E. Witten, Supersymmetry and Morse theory, J. Diff. Geom. 17 (1982) 661, http://projecteuclid.org/euclid.jdg/1214437492.

[60] E. Witten, Elliptic Genera and Quantum Field Theory, Commun. Math. Phys. 109 (1987) 525 [INSPIRE].

[61] N.A. Nekrasov, Seiberg-Witten prepotential from instanton counting, Adv. Theor. Math. Phys. 7 (2003) 831 [hep-th/0206161] [INSPIRE].

[62] N. Nekrasov and A. Okounkov, Seiberg-Witten theory and random partitions, Prog. Math. 244 (2006) 525 [hep-th/0306238] [INSPIRE].

[63] N.A. Nekrasov and S.L. Shatashvili, Quantization of Integrable Systems and Four Dimensional Gauge Theories, in Proceedings, 16th International Congress on Mathematical Physics (ICMP09): Prague, Czech Republic, August 3-8, 2009, pp. 265-289, arXiv:0908.4052 [INSPIRE].

[64] F. Verstraete, V. Murg and J. Cirac, Matrix product states, projected entangled pair states, and variational renormalization group methods for quantum spin systems, Adv. Phys. $\mathbf{5 7}$ (2008) 143.

[65] M. Ünsal and L.G. Yaffe, Large- $N$ volume independence in conformal and confining gauge theories, JHEP 08 (2010) 030 [arXiv:1006.2101] [INSPIRE].

[66] A. Cherman, S. Sen, M.L. Wagman and L.G. Yaffe, Exponential reduction of finite volume effects with twisted boundary conditions, Phys. Rev. D 95 (2017) 074512 [arXiv: 1612.00403] [INSPIRE].

[67] G. Basar, A. Cherman and D.A. McGady, Bose-Fermi Degeneracies in Large $N$ Adjoint QCD, JHEP 07 (2015) 016 [arXiv: 1409.1617] [INSPIRE].

[68] D.J. Gross and Y. Kitazawa, A Quenched Momentum Prescription for Large $N$ Theories, Nucl. Phys. B 206 (1982) 440 [inSPIRE].

[69] A. Gonzalez-Arroyo and M. Okawa, The Twisted Eguchi-Kawai Model: A Reduced Model for Large N Lattice Gauge Theory, Phys. Rev. D 27 (1983) 2397 [INSPIRE].

[70] A. Gonzalez-Arroyo and M. Okawa, A Twisted Model for Large N Lattice Gauge Theory, Phys. Lett. B 120 (1983) 174 [INSPIRE].

[71] A. Gonzalez-Arroyo and M. Okawa, Large $N$ reduction with the Twisted Eguchi-Kawai model, JHEP 07 (2010) 043 [arXiv: 1005.1981] [inSPIRE].

[72] A.J. Macfarlane, Solution of the Schrödinger equation of the complex manifold $C P^{n}$, J. Phys. A 36 (2003) 9689.

[73] A.M. Perelomov, Chiral models: geometrical aspects, Phys. Rept. 146 (1987) 135 [InSPIRE].

[74] W.J. Zakrzewski, Classical solutions of two-dimensional Grassmannian models, J. Geom. Phys. 1 (1984) 39 [INSPIRE].

[75] A. Yu. Morozov, A.M. Perelomov and M.A. Shifman, Exact Gell-Mann-Low Function of supersymmetric Kähler $\sigma$-models, Nucl. Phys. B 248 (1984) 279 [INSPIRE].

[76] E. Witten, Instantons, the Quark Model and the 1/n Expansion, Nucl. Phys. B 149 (1979) 285 [INSPIRE]. 
[77] I. Affleck, The Role of Instantons in Scale Invariant Gauge Theories, Nucl. Phys. B 162 (1980) 461 [INSPIRE].

[78] T. Sulejmanpasic and Y. Tanizaki, C-P-T anomaly matching in bosonic quantum field theory and spin chains, Phys. Rev. B 97 (2018) 144201 [arXiv:1802.02153] [INSPIRE].

[79] A. Kapustin and N. Seiberg, Coupling a QFT to a TQFT and Duality, JHEP 04 (2014) 001 [arXiv: 1401.0740] [INSPIRE].

[80] N.D. Mermin and H. Wagner, Absence of ferromagnetism or antiferromagnetism in one-dimensional or two-dimensional isotropic Heisenberg models, Phys. Rev. Lett. 17 (1966) 1133 [INSPIRE].

[81] S.R. Coleman, There are no Goldstone bosons in two-dimensions, Commun. Math. Phys. 31 (1973) 259 [INSPIRE].

[82] X. Chen, Z.-C. Gu and X.-G. Wen, Classification of gapped symmetric phases in one-dimensional spin systems, Phys. Rev. B 83 (2011) 035107 [arXiv:1008.3745].

[83] F.D.M. Haldane, Continuum dynamics of the 1-D Heisenberg antiferromagnetic identification with the $O(3)$ nonlinear $\sigma$-model, Phys. Lett. A 93 (1983) 464 [INSPIRE].

[84] F.D.M. Haldane, Nonlinear field theory of large spin Heisenberg antiferromagnets. Semiclassically quantized solitons of the one-dimensional easy Axis Neel state, Phys. Rev. Lett. 50 (1983) 1153 [INSPIRE].

[85] A. Cherman, S. Sen, M. Ünsal, M.L. Wagman and L.G. Yaffe, Order parameters and color-flavor center symmetry in QCD, Phys. Rev. Lett. 119 (2017) 222001 [arXiv: 1706.05385] [INSPIRE].

[86] D. Tong, The moduli space of BPS domain walls, Phys. Rev. D 66 (2002) 025013 [hep-th/0202012] [INSPIRE].

[87] F. Bruckmann, Instanton constituents in the $O(3)$ model at finite temperature, Phys. Rev. Lett. 100 (2008) 051602 [arXiv: 0707.0775] [INSPIRE].

[88] W. Brendel, F. Bruckmann, L. Janssen, A. Wipf and C. Wozar, Instanton constituents and fermionic zero modes in twisted CP**n models, Phys. Lett. B 676 (2009) 116 [arXiv: 0902.2328] [INSPIRE].

[89] I. Affleck and E.H. Lieb, A Proof of Part of Haldane's Conjecture on Spin Chains, Lett. Math. Phys. 12 (1986) 57 [INSPIRE].

[90] I. Affleck, T. Kennedy, E.H. Lieb and H. Tasaki, Rigorous Results on Valence Bond Ground States in Antiferromagnets, Phys. Rev. Lett. 59 (1987) 799 [InSPIRE].

[91] F.D.M. Haldane, Model for a Quantum Hall Effect without Landau Levels: Condensed-Matter Realization of the 'Parity Anomaly', Phys. Rev. Lett. 61 (1988) 2015 [InSPIRE]. 